\title{
Parametric decay instability near the upper hybrid resonance in magnetically confined
} fusion plasmas

Hansen, Søren Kjer; Nielsen, Stefan Kragh; Salewski, Mirko; Pedersen, Morten Stejner; Stober, J.

Published in:

Plasma Physics and Controlled Fusion

Link to article, DOI:

10.1088/1361-6587/aa7978

Publication date:

2017

Document Version

Peer reviewed version

Link back to DTU Orbit

Citation (APA):

Hansen, S. K., Nielsen, S. K., Salewski, M., Pedersen, M. S., \& Stober, J. (2017). Parametric decay instability near the upper hybrid resonance in magnetically confined fusion plasmas. Plasma Physics and Controlled Fusion, 59(10), [105006 ]. https://doi.org/10.1088/1361-6587/aa7978

\section{General rights}

Copyright and moral rights for the publications made accessible in the public portal are retained by the authors and/or other copyright owners and it is a condition of accessing publications that users recognise and abide by the legal requirements associated with these rights.

- Users may download and print one copy of any publication from the public portal for the purpose of private study or research.

- You may not further distribute the material or use it for any profit-making activity or commercial gain

- You may freely distribute the URL identifying the publication in the public portal 


\title{
Parametric Decay Instability near the Upper Hybrid Resonance in Magnetically Confined Fusion Plasmas
}

\author{
S K Hansen ${ }^{1,2}$, S K Nielsen ${ }^{2}$, M Salewski ${ }^{2}$, M Stejner ${ }^{2}$, J \\ Stober $^{1}$ and the ASDEX Upgrade team ${ }^{1}$ \\ 1 Max-Planck-Institut für Plasmaphysik, Boltzmannstraße, D-85748 Garching bei \\ München, Germany \\ 2 Department of Physics, Technical University of Denmark, Fysikvej, DK-2800 Kgs. \\ Lyngby, Denmark \\ E-mail: Soeren.Kjer.Hansen@ipp.mpg.de
}

\begin{abstract}
In this paper we investigate parametric decay of an electromagnetic pump wave into two electrostatic daughter waves, particularly an X-mode pump wave decaying into a warm upper hybrid wave (a limit of an electron Bernstein wave) and a warm lower hybrid wave. We describe the general theory of the above parametric decay instability (PDI), unifying earlier treatments, and show that it may occur in underdense and weakly overdense plasmas. The PDI theory is used to explain the anomalous sidebands observed in collective Thomson scattering (CTS) spectra at the ASDEX Upgrade tokamak. The theory may also account for similar observations during CTS experiments in stellarators, as well as in some 1st harmonic electron cyclotron resonance and $\mathrm{O}-\mathrm{X}-\mathrm{B}$ heating experiments.
\end{abstract}

Keywords: PDI, UHR, UH, LH, CTS, ECRH, Electron Bernstein Wave

\section{Introduction}

A parametric decay instability (PDI) is the result of three-wave interactions by which an incident (pump) wave decays to two daughter waves. PDIs may occur in some media under the influence of pump radiation with sufficiently high energy density, e.g. in plasmas heated/diagnosed by gyrotron radiation. Energy and momentum conservation in the three-wave processes impose the selection rules that the sum of the daughter wave frequencies and wave vectors should be that of the pump wave, resulting in frequencyshifted radiation which interferes with millimetre-wave diagnostics such as electron cyclotron (EC) emission and collective Thomson scattering (CTS). This is of particular concern in future fusion reactors which cannot be easily diagnosed by other means. In this work we focus on parametric decay of an electromagnetic pump wave into two electrostatic daughter waves, specifically the case of an X-mode pump wave decaying into a warm upper hybrid (UH) wave (a limiting form of an electron Bernstein wave) and a warm lower hybrid (LH) wave near the upper hybrid resonance (UHR) of the pump wave. We generalise the results of $[1,3]$ and discuss their relation to other treatments [4,5]. The 
main motivation is observations of strong PDI-like sidebands during CTS experiments at the ASDEX Upgrade tokamak [6] and the LHD stellarator [7,8].

Early PDI theory for unmagnetised and magnetised plasmas was pioneered by [9, 10 and extended to include collisional effects, which usually determine the PDI threshold in homogeneous plasmas, by [11]. Piliya and Rosenbluth separately showed that the PDI threshold could be significantly higher for an inhomogeneous plasma due to convective losses of the daughter waves from the region where the PDI selection rules are satisfied [12 14]. The above results indicate that PDIs are only likely to occur near resonances, e.g. the UHR, and cut-offs of the pump radiation at the gyrotron powers available in typical experiments, due to field enhancement effects in these regions [15]. An important exception to this rule, which has received some attention recently, occurs in regions of non-monotonic plasma profiles where the daughter waves may become trapped, reducing the PDI threshold to a level closer to that of a homogeneous plasma [16]; the backscattering instability is another example of this type [17.

PDIs near the UHR have been studied theoretically by a number of authors. The first work on direct parametric decay of an electromagnetic pump wave into warm UH waves and LH waves was [4], which found the growth rate of this PDI in the limit of long daughter wave wavelengths relative to the ion Larmor radius, a condition rarely satisfied in magnetically confined fusion plasmas. Direct parametric decay of an electromagnetic pump wave into a warm UH wave and a strongly damped low-frequency electrostatic quasi-mode, which is particularly relevant in tokamaks, was first considered by Ott, Hui and $\mathrm{Chu}$ [18 who concluded that it would be unlikely to occur for gyrotron radiation. However, their treatment neglected field enhancement effects and used a non-standard parametric dispersion relation. Using the standard parametric dispersion relation, and accounting roughly for field enhancement near the UHR, Porkolab [2] showed that the PDI in which an X-mode pump wave decays into a warm UH wave and an LH quasi-mode could occur during 1st harmonic X-mode EC resonance heating (ECRH) on the Versator II tokamak, as later confirmed experimentally [3]. The theoretical part of the present paper mainly deals with generalising the results from [1-3]. Further theoretical work on direct parametric decay of electromagnetic pump waves to electrostatic daughter waves near the UHR is presented in $[5,19,20]$. Other theoretical papers focus on parametric decay of warm UH waves, generated by linear mode conversion, into other warm UH waves and electrostatic low-frequency waves [17,21] or into electromagnetic high-frequency waves and electrostatic low-frequency $(\mathrm{LH})$ waves 22,23 . Many of the theoretical results have been reproduced in numerical simulations 24 27.

A PDI at the UHR was first observed in mercury vapour tube discharges through the excitation of LH oscillations by sufficiently strong microwave radiation 28]. Similar observations were reported for linear hot-cathode helium discharges [29] and the FM-I spherator [30]. This type of PDI was also observed in connection with 1st harmonic ECRH of optically thin plasmas in the Versator II, FT-1 and TCA tokamaks [3, 31, 32, as well as in the Wendelstein 7-A stellarator [33, 34]. These results were reviewed by 35,36 . In ionospheric modification experiments, the presence of a so-called down- 
shifted maximum feature in the stimulated electromagnetic emission spectra has been attributed to a related PDI [23]. PDIs near the UHR involving LH daughter waves have additionally been used to confirm the occurrence of O-X-B heating in overdense plasmas of the Wendelstein 7-AS stellarator [37, 38] and the MAST spherical tokamak [39].

This paper is arranged as follows. In Section 2, we review the general theory of PDIs for electromagnetic pump waves decaying into electrostatic daughter waves in a homogeneous plasma and its generalisation to weakly inhomogeneous plasmas, providing a unified treatment and re-examining the existing literature. In Section 3, we specialise to PDIs near the UHR, investigating the conditions under which these may occur and generalising the theories of parametric decay into warm UH and warm LH waves of [1 3$]$. Finally, we investigate the above PDI in ASDEX Upgrade and compare the theoretical predictions with experimentally observed CTS spectra in Section 4.

\section{PDI Theory}

In this section we present the basic theory of parametric decay of an electromagnetic pump wave to electrostatic daughter waves in a magnetised plasma. Apart from allowing us to introduce the concepts needed when discussing PDIs near the UHR, this is motivated by the existence of large number of different, not always consistent, treatments in the literature, e.g. 44, 5, 10, 11, 18, 20, 35, 40], which seem to warrant some discussion and clarification. Our treatment follows that of [1] quite closely.

The main parametric three-wave processes are decay of the pump wave into two daughter waves and scattering of the pump wave by one of the daughter waves, as illustrated in figure 1; PDIs are associated with the decay process, but in order to interpret PDI spectra, and to have a complete theory, it is necessary to consider the scattering process as well. Processes involving more than three waves may also occur, but these represent higher-order effects. Energy and momentum conservation in the three-wave processes impose selection rules for the frequencies, $\omega$, and wave vectors, $\mathbf{k}$, of the involved waves,

$$
\omega_{2}=\omega_{0}-\omega_{1}, \quad \mathbf{k}_{2}=\mathbf{k}_{0}-\mathbf{k}_{1}, \omega_{3}=\omega_{0}+\omega_{1}, \mathbf{k}_{3}=\mathbf{k}_{0}+\mathbf{k}_{1},
$$

where subscript 0 refers to the pump wave, subscript 1 refers to the low-frequency daughter wave, and subscripts 2 and 3 refer to the down- and up-shifted high-frequency daughter waves, respectively. A PDI will generally only occur if the selection rules can be satisfied by linear modes of the plasma, as the pump power necessary for a PDI to occur under normal circumstances increases with the damping rate of the daughter waves; however, in some cases the low-frequency daughter wave may be a heavily damped quasi-mode. The above considerations lead to an idealised PDI frequency spectrum, seen in figure 2, which is a hallmark of PDIs. 

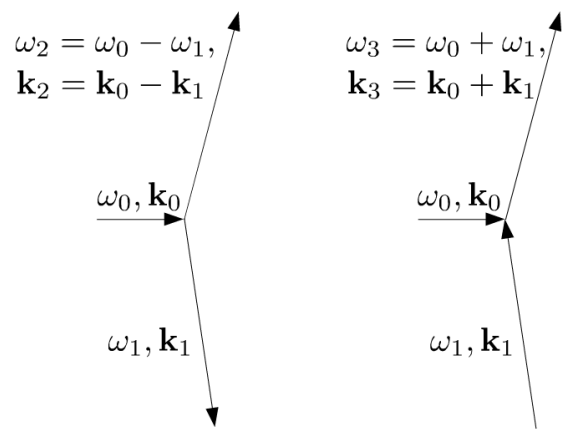

Figure 1. Diagrams showing the most important parametric three-wave processes; to the left, decay of the pump wave $\left(\omega_{0}, \mathbf{k}_{0}\right)$ into a low-frequency wave $\left(\omega_{1}, \mathbf{k}_{1}\right)$ and a down-shifted high-frequency wave $\left(\omega_{2}=\omega_{0}-\right.$ $\omega_{1}, \mathbf{k}_{2}=\mathbf{k}_{0}-\mathbf{k}_{1}$ ); to the right, scattering of the pump wave by a low-frequency wave exciting an up-shifted high-frequency wave $\left(\omega_{3}=\omega_{0}+\omega_{1}, \mathbf{k}_{3}=\mathbf{k}_{0}+\mathbf{k}_{1}\right)$.

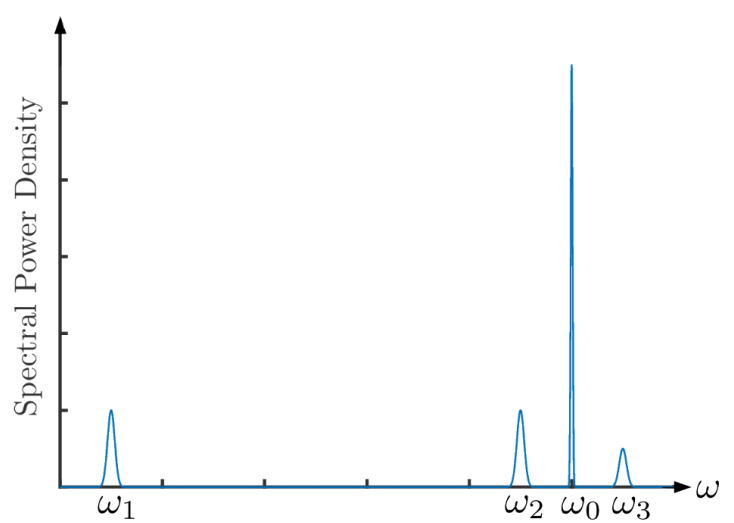

Figure 2. Idealised frequency power spectrum excited by a PDI. Apart from the pump peak $\left(\omega_{0}\right)$, a low-frequency daughter peak $\left(\omega_{1}\right)$ and high-frequency daughter peaks, one down-shifted $\left(\omega_{2}\right)$ and one up-shifted $\left(\omega_{3}\right)$, occur. Peaks are generally observed when $\omega_{1}$, $\omega_{2}$ and $\omega_{3}$ coincide with linear modes.

\subsection{Motion of an Electromagnetically Driven Plasma Particle}

To describe the PDI of interest we first consider the motion of a single plasma particle with mass $m_{\sigma}$ and charge $q_{\sigma}$, located at position $\mathbf{r}(t)$ and moving with a velocity $\mathbf{v}(t)=\mathrm{d} \mathbf{r}(t) / \mathrm{d} t$ at time $t$. The particle is acted on by a steady homogeneous magnetic field $\mathbf{B}$ and a plane electromagnetic pump wave with an electric field $\operatorname{Re}\left[\mathbf{E}_{0} \mathrm{e}^{\mathrm{i} \mathbf{k}_{0} \cdot \mathbf{r}(t)-\omega_{0} t}\right]$ and a magnetic field $\operatorname{Re}\left[\mathbf{B}_{0} \mathrm{e}^{\mathrm{i} \mathbf{k}_{0} \cdot \mathbf{r}(t)-\omega_{0} t}\right]$. We assume the particle to be non-relativistic and invoke the dipole approximation for the electromagnetic pump wave, $\mathbf{k}_{0} \approx \mathbf{0}$, which allows us to neglect the effect of the wave magnetic field and to write the wave electric field as $\operatorname{Re}\left(\mathbf{E}_{0} \mathrm{e}^{-\mathrm{i} \omega_{0} t}\right)$; the dipole approximation is generally permissible due to the high phase velocity (long wavelength) of the electromagnetic pump wave compared with the electrostatic daughter waves, but may be relaxed if necessary [19]. Taking the background magnetic field along the $z$-axis, $\mathbf{B}=B \mathbf{e}_{z}$, the equation of motion becomes

$$
\frac{\mathrm{d} \mathbf{v}(t)}{\mathrm{d} t}=\omega_{c \sigma} \mathbf{v}(t) \times \mathbf{e}_{z}+\operatorname{Re}\left(\frac{q_{\sigma} \mathbf{E}_{0}}{m_{\sigma}} \mathrm{e}^{-\mathrm{i} \omega_{0} t}\right),
$$

where $\omega_{c \sigma}=q_{\sigma} B / m_{\sigma}$ is the cyclotron frequency (with sign) of species $\sigma$. (2) is a set of inhomogeneous, linear, ordinary differential equations, the general solution of which is a given by a particular solution of the inhomogeneous problem and a general solution of the homogeneous problem. The general homogeneous solution is cyclotron motion of the particle in a constant magnetic field, i.e. the characteristics used for determining the linear response of a collisionless plasma. PDIs originate from the particular inhomogeneous solution which can be found by substituting the ansatz 
$\mathbf{v}_{p \sigma}(t)=\operatorname{Re}\left(\mathbf{v}_{p 0 \sigma} \mathrm{e}^{-\mathrm{i} \omega_{0} t}\right)$ into (2), solving for $\mathbf{v}_{p 0 \sigma}$, and integrating once more with respect to $t$, looking for a solution of the form $\mathbf{r}_{p \sigma}(t)=\operatorname{Re}\left(\mathbf{r}_{p 0 \sigma} \mathrm{e}^{-\mathrm{i} \omega_{0} t}\right)$,

$\mathbf{r}_{p \sigma}(t)=\operatorname{Re}\left(\frac{\mathrm{i} \mathbf{v}_{p 0 \sigma}}{\omega_{0}} \mathrm{e}^{-\mathrm{i} \omega_{0} t}\right), \mathbf{v}_{p 0 \sigma}=\frac{q_{\sigma}}{m_{\sigma}}\left[\begin{array}{c}\left(\mathrm{i} \omega_{0} E_{0 x}-\omega_{c \sigma} E_{0 y}\right) /\left(\omega_{0}^{2}-\omega_{c \sigma}^{2}\right) \\ \left(\omega_{c \sigma} E_{0 x}+\mathrm{i} \omega_{0} E_{0 y}\right) /\left(\omega_{0}^{2}-\omega_{c \sigma}^{2}\right) \\ E_{0 z} / \omega_{0}\end{array}\right]$.

The free energy responsible for driving PDIs is the kinetic energy associated with this motion. The complex displacement vector, $\mathbf{r}_{p 0 \sigma}=\mathbf{i} \mathbf{v}_{p 0 \sigma} / \omega_{0}$, agrees with the one given in (3) of [19] for electrons.

\subsection{Kinetic Theory of PDIs in Homogeneous Plasmas}

The goal of this subsection is to derive the temporal PDI growth rate, $\gamma$, for the case of small amplitude electrostatic daughter waves in a homogeneous, charge neutral plasma. This problem has previously been considered by a number of authors, e.g. in [4, 5, 10, 11, 18, 35, 40], and we shall point out when our results agree/disagree with some this earlier work. PDIs with electrostatic daughter waves are governed by the Boltzmann-Poisson system with external fields $\mathbf{B}=B \mathbf{e}_{z}$ and $\operatorname{Re}\left(\mathbf{E}_{0} \mathrm{e}^{-\mathrm{i} \omega_{0} t}\right)$,

$$
\begin{aligned}
\frac{\partial f_{\sigma}(\mathbf{r}, \mathbf{v}, t)}{\partial t}+\mathbf{v} \cdot \frac{\partial f_{\sigma}(\mathbf{r}, \mathbf{v}, t)}{\partial \mathbf{r}}+ & {\left[-\frac{q_{\sigma}}{m_{\sigma}} \frac{\partial \phi(\mathbf{r}, t)}{\partial \mathbf{r}}+\omega_{c \sigma} \mathbf{v} \times \mathbf{e}_{z}\right.} \\
& \left.+\operatorname{Re}\left(\frac{q_{\sigma} \mathbf{E}_{0}}{m_{\sigma}} \mathrm{e}^{-\mathrm{i} \omega_{0} t}\right)\right] \cdot \frac{\partial f_{\sigma}(\mathbf{r}, \mathbf{v}, t)}{\partial \mathbf{v}}=\left[\frac{\partial f_{\sigma}(\mathbf{r}, \mathbf{v}, t)}{\partial t}\right]_{\text {col }}, \\
\frac{\partial}{\partial \mathbf{r}} \cdot \frac{\partial \phi(\mathbf{r}, t)}{\partial \mathbf{r}}= & -\frac{1}{\epsilon_{0}} \sum_{\sigma} q_{\sigma} n_{\sigma}(\mathbf{r}, t)
\end{aligned}
$$

where $f_{\sigma}(\mathbf{r}, \mathbf{v}, t)$ is the distribution function of species $\sigma, n_{\sigma}(\mathbf{r}, t)=\int_{\text {all } \mathbf{v}} f_{\sigma}(\mathbf{r}, \mathbf{v}, t) \mathrm{d} \mathbf{v}$ is the number density of species $\sigma, \phi(\mathbf{r}, t)$ is the electrostatic potential associated with the daughter waves, $\left[\partial f_{\sigma}(\mathbf{r}, \mathbf{v}, t) / \partial t\right]_{\text {col }}$ is the collision operator of species $\sigma$, and $\epsilon_{0}$ is the vacuum permittivity. Evidently, the pump wave introduces an explicit dependence on $t$ which makes the above equations more complicated than the usual BoltzmannPoisson system in a magnetised plasma. However, by going into frames oscillating with the velocity induced by the pump wave for each species $\sigma, \mathbf{v}_{p \sigma}(t)$, inertial forces will cancel the explicitly $t$-dependent pump term, $\operatorname{Re}\left[\left(q_{\sigma} \mathbf{E}_{0} / m_{\sigma}\right) \mathrm{e}^{-\mathrm{i} \omega_{0} t}\right]$. In these frames the position is defined as $\mathbf{x}=\mathbf{r}-\mathbf{r}_{p \sigma}(t)$, the velocity is defined as $\mathbf{u}=\mathbf{v}-\mathbf{v}_{p \sigma}(t)$, and the Boltzmann equation becomes

$$
\begin{aligned}
\frac{\partial F_{\sigma}(\mathbf{x}, \mathbf{u}, t)}{\partial t}+\mathbf{u} \cdot \frac{\partial F_{\sigma}(\mathbf{x}, \mathbf{u}, t)}{\partial \mathbf{x}}+ & {\left[-\frac{q_{\sigma}}{m_{\sigma}} \frac{\partial \Phi_{\sigma}(\mathbf{x}, t)}{\partial \mathbf{x}}\right.} \\
& \left.+\omega_{c \sigma} \mathbf{u} \times \mathbf{e}_{z}\right] \cdot \frac{\partial F_{\sigma}(\mathbf{x}, \mathbf{u}, t)}{\partial \mathbf{u}}=\left[\frac{\partial F_{\sigma}(\mathbf{x}, \mathbf{u}, t)}{\partial t}\right]_{\mathrm{col}},
\end{aligned}
$$

where $F_{\sigma}(\mathbf{x}, \mathbf{u}, t)=f_{\sigma}(\mathbf{r}, \mathbf{v}, t)$ is the distribution function, $\Phi_{\sigma}(\mathbf{x}, t)=\phi(\mathbf{r}, t)$ is the electrostatic potential associated with the daughter waves, and $\left[\partial F_{\sigma}(\mathbf{x}, \mathbf{u}, t) / \partial t\right]_{\mathrm{col}}=$ $\left[\partial f_{\sigma}(\mathbf{r}, \mathbf{v}, t) / \partial t\right]_{\mathrm{col}}$ is the collision operator, all in the frame of species $\sigma$. This is a standard form of the Boltzmann equation used in the kinetic description of electrostatic 
waves. Since we are assuming the amplitude of the daughter waves to be small and the unperturbed plasma to be homogeneous and charge neutral, we can carry through an order 1 perturbation analysis for electrostatic waves in which we take $F_{\sigma}(\mathbf{x}, \mathbf{u}, t)=F_{\sigma}^{(0)}(\mathbf{u})+F_{\sigma}^{(1)}(\mathbf{x}, \mathbf{u}, t), \Phi_{\sigma}(\mathbf{x}, t)=\Phi_{\sigma}^{(1)}(\mathbf{x}, t)$, and neglect products of the perturbation terms with superscript (1). The general result of this procedure is a local, linear relation between the Fourier-Laplace transforms of the perturbed number density $N_{\sigma}^{(1)}(\mathbf{x}, t)=\int_{\text {all } \mathbf{u}} F_{\sigma}^{(1)}(\mathbf{x}, \mathbf{u}, t) \mathrm{d} \mathbf{u}$ and $\Phi_{\sigma}(\mathbf{x}, t)$. Defining the Fourier-Laplace transform as $\tilde{g}(\mathbf{k}, \omega)=\int_{\text {all } \mathbf{x}}\left[\int_{0}^{\infty} g(\mathbf{x}, t) \mathrm{e}^{\mathrm{i} \omega t-\mathbf{i} \cdot \mathbf{x}} \mathrm{d} t\right] \mathrm{d} \mathbf{x}$, with $\mathbf{k}$ being a real wave vector and $\omega$ being a complex frequency related to a daughter wave mode, we may thus write

$$
q_{\sigma} \tilde{N}_{\sigma}^{(1)}(\mathbf{k}, \omega)=-\epsilon_{0} k^{2} \chi_{\sigma}(\mathbf{k}, \omega) \tilde{\Phi}_{\sigma}(\mathbf{k}, \omega),
$$

where $\chi_{\sigma}(\mathbf{k}, \omega)$ is the linear susceptibility of species $\sigma$ for the mode characterised by $(\mathbf{k}, \omega)$; in writing this, we have set the perturbations to zero at $t=0$. The temporal growth rate of the PDI in a homogeneous plasma is given by the imaginary part of $\omega, \gamma=\operatorname{Im}(\omega)$, and is the quantity in which we are ultimately interested. $\chi_{\sigma}(\mathbf{k}, \omega)$ is determined by $F_{\sigma}^{(0)}(\mathbf{u})$ and $\left[\partial F_{\sigma}(\mathbf{x}, \mathbf{u}, t) / \partial t\right]_{\text {col }}$, which are considered to be a Maxwellian distribution and a particle conserving Krook collision operator in this paper:

$F_{\sigma}^{(0)}(\mathbf{u})=\frac{N_{\sigma}^{(0)}}{\pi^{3 / 2} v_{T \sigma}^{3}} \mathrm{e}^{-u^{2} / v_{T \sigma}^{2}}, \quad\left[\frac{\partial F_{\sigma}(\mathbf{x}, \mathbf{u}, t)}{\partial t}\right]_{\mathrm{col}}=\nu_{\sigma}\left[\frac{N_{\sigma}^{(1)}(\mathbf{x}, t)}{N_{\sigma}^{(0)}} F_{\sigma}^{(0)}(\mathbf{u})-F_{\sigma}^{(1)}(\mathbf{x}, \mathbf{u}, t)\right]$,

where $N_{\sigma}^{(0)}$ is the unperturbed number density of species $\sigma, v_{T \sigma}=\sqrt{2 T_{\sigma} / m_{\sigma}}$ is the thermal velocity of species $\sigma$, with temperature $T_{\sigma}$, and $\nu_{\sigma}$ is a phenomenological collision frequency of species $\sigma$, which we shall equate with the Coulomb collision frequency of species $\sigma$ as is customary 41 . For the above $F_{\sigma}^{(0)}(\mathbf{u})$ and $\left[\partial F_{\sigma}(\mathbf{x}, \mathbf{u}, t) / \partial t\right]_{\mathrm{col}}, \chi_{\sigma}(\mathbf{k}, \omega)$ becomes 11 ,

$$
\chi_{\sigma}(\mathbf{k}, \omega)=\frac{2 \omega_{p \sigma}^{2}}{k^{2} v_{T \sigma}^{2}} \frac{1+\frac{\omega+\mathrm{i} \nu_{\sigma}}{k_{z} v_{T \sigma}}}{1+\frac{\mathrm{i} \nu_{\sigma}}{k_{z} v_{T \sigma}}} \sum_{n=-\infty}^{\infty} I_{n=-\infty}^{\infty} I_{n}\left(k_{\perp}^{2} r_{L \sigma}^{2} r_{L \sigma}^{2}\right) \mathrm{e}^{-k_{\perp}^{2} r_{L \sigma}^{2} r_{L \sigma}^{2} Z\left(\frac{\omega+\mathrm{i} \nu_{\sigma}-n \omega_{c \sigma}}{k_{z} v_{T \sigma}}\right)},
$$

with $k_{\perp}^{2}=k_{x}^{2}+k_{y}^{2}$ being the wave number perpendicular to $\mathbf{B}, \omega_{p \sigma}^{2}=q_{\sigma}^{2} N_{\sigma}^{(0)} /\left(\epsilon_{0} m_{\sigma}\right)$ being the plasma frequency of species $\sigma, r_{L \sigma}=v_{T \sigma} /\left(\sqrt{2}\left|\omega_{c \sigma}\right|\right)$ defining the Larmor radius of species $\sigma, I_{n}$ being the order $n$ modified Bessel function of the 1st kind, and $Z$ being the Fried-Conte plasma dispersion function. In case of the ions we shall also employ $\chi_{\sigma}(\mathbf{k}, \omega)$ for an unmagnetised plasma with the same $F_{\sigma}^{(0)}(\mathbf{u})$ and $\left[\partial F_{\sigma}(\mathbf{x}, \mathbf{u}, t) / \partial t\right]_{\mathrm{col}}[41$,

$$
\chi_{\sigma}(\mathbf{k}, \omega)=\frac{2 \omega_{p \sigma}^{2}}{k^{2} v_{T \sigma}^{2}} \frac{1+\frac{\omega+\mathrm{i} \nu_{\sigma}}{k v_{T \sigma}} Z\left(\frac{\omega+\mathrm{i} \nu_{\sigma}}{k v_{T \sigma}}\right)}{1+\frac{\mathrm{i} \nu_{\sigma}}{k v_{T \sigma}} Z\left(\frac{\omega+\mathrm{i} \nu_{\sigma}}{k v_{T \sigma}}\right)}
$$

the justification for this is discussed in Subsection 3.2.

The problem now is to express $\tilde{\Phi}_{\sigma}(\mathbf{k}, \omega)$, which is evaluated in a different frame for each species, in terms of the potential in the lab frame. This may be done by using the 
definition of the Fourier-Laplace transform to write,

$\tilde{\Phi}_{\sigma}(\mathbf{k}, \omega)=\int_{\text {all } \mathbf{x}}\left[\int_{0}^{\infty} \Phi_{\sigma}(\mathbf{x}, t) \mathrm{e}^{\mathrm{i} \omega t-\mathrm{i} \mathbf{k} \cdot \mathbf{x}} \mathrm{d} t\right] \mathrm{d} \mathbf{x}=\int_{\text {all } \mathbf{r}}\left[\int_{0}^{\infty} \phi(\mathbf{r}, t) \mathrm{e}^{\mathrm{i} \omega t+\mathrm{i} \mathbf{k} \cdot \mathbf{r}_{p \sigma}(t)} \mathrm{d} t\right] \mathrm{e}^{-\mathrm{i} \mathbf{k} \cdot \mathbf{r}} \mathrm{d} \mathbf{r}$,

which reduces the problem to evaluating $\mathrm{e}^{\mathrm{ik} \cdot \mathbf{r}_{p \sigma}(t)}$ and the Fourier-Laplace integrals. Evaluation of $\mathrm{e}^{\mathrm{i} \mathbf{k} \cdot \mathbf{r}_{p \sigma}(t)}$ is facilitated by noting that, from (3) and the fact that $\mathbf{k}$ is real, $\mathbf{k} \cdot \mathbf{r}_{p \sigma}(t)=\operatorname{Re}\left[\mathrm{i}\left(\mathbf{k} \cdot \mathbf{v}_{p 0 \sigma} / \omega_{0}\right) \mathrm{e}^{-\mathrm{i} \omega_{0} t}\right]=\mu_{\sigma} \sin \left(\omega_{0} t-\beta_{\sigma}\right)$, where $\mu_{\sigma}$ and $\beta_{\sigma}$ are the amplitude and phase angle of $\mathbf{k} \cdot \mathbf{v}_{p 0 \sigma} / \omega_{0}$, respectively. Using a Fourier series identity, we then find $\mathrm{e}^{\mathrm{i} \mathbf{k} \cdot \mathbf{r}_{p \sigma}(t)}=\mathrm{e}^{\mathrm{i} \mu_{\sigma} \sin \left(\omega_{0} t-\beta_{\sigma}\right)}=\sum_{n=-\infty}^{\infty} J_{n}\left(\mu_{\sigma}\right) \mathrm{e}^{\mathrm{i} n\left(\omega_{0} t-\beta_{\sigma}\right)}$, where $J_{n}$ is the order $n$ Bessel function of the 1st kind. With this, the Fourier-Laplace integrals of (11) may be evaluated, and (7) written as

$$
q_{\sigma} \tilde{N}_{\sigma}^{(1)}(\mathbf{k}, \omega)=-\epsilon_{0} k^{2} \chi_{\sigma}(\mathbf{k}, \omega) \sum_{n=-\infty}^{\infty} J_{n}\left(\mu_{\sigma}\right) \mathrm{e}^{-\mathrm{i} n \beta_{\sigma}} \tilde{\phi}\left(\mathbf{k}, \omega+n \omega_{0}\right) .
$$

By Fourier-Laplace transforming the Poisson equation with a charge neutral unperturbed plasma, $(\partial / \partial \mathbf{r}) \cdot[\partial \phi(\mathbf{r}, t) / \partial \mathbf{r}]=-\left(1 / \epsilon_{0}\right) \sum_{\sigma} q_{\sigma} N_{\sigma}^{(1)}\left(\mathbf{r}-\mathbf{r}_{p \sigma}(t), t\right)$, we similarly find

$$
\tilde{\phi}(\mathbf{k}, \omega)=\frac{1}{\epsilon_{0} k^{2}} \sum_{\sigma} \sum_{n=-\infty}^{\infty} J_{n}\left(-\mu_{\sigma}\right) \mathrm{e}^{-\mathrm{i} n \beta_{\sigma}} q_{\sigma} \tilde{N}_{\sigma}^{(1)}\left(\mathbf{k}, \omega+n \omega_{0}\right) .
$$

(12) and (13) constitute a set of linear equations describing the coupling between various electrostatic Fourier-Laplace modes due to the pump wave. These equations form a general basis for the theory of PDIs with small amplitude electrostatic daughter waves in a homogeneous plasma within the dipole approximation. They were also derived or used as a starting point by [11,35, 40; 11 generalised the result to inhomogeneous plasmas. $\mu_{\sigma}=\left|\mathbf{k} \cdot \mathbf{v}_{p 0 \sigma}\right| / \omega_{0}$ describes the coupling strength between different FourierLaplace modes and is a central quantity in PDI theory; inserting $\mathbf{v}_{p 0 \sigma}$ from (3),

$\mu_{\sigma}=\frac{\left|q_{\sigma}\right|}{m_{\sigma} \omega_{0}^{2}} \sqrt{\frac{\left[\frac{\operatorname{Im}\left(k_{x} E_{0 x}+k_{y} E_{0 y}\right)+\left(\omega_{c \sigma} / \omega_{0}\right) \operatorname{Re}\left(k_{x} E_{0 y}-k_{y} E_{0 x}\right)}{1-\left(\omega_{c \sigma} / \omega_{0}\right)^{2}}+k_{z} \operatorname{Im}\left(E_{0 z}\right)\right]^{2}+}{\left[\frac{\operatorname{Re}\left(k_{x} E_{0 x}+k_{y} E_{0 y}\right)-\left(\omega_{c \sigma} / \omega_{0}\right) \operatorname{Im}\left(k_{x} E_{0 y}-k_{y} E_{0 x}\right)}{1-\left(\omega_{c \sigma} / \omega_{0}\right)^{2}}+k_{z} \operatorname{Re}\left(E_{0 z}\right)\right]^{2}},}$

which shows the general proportionality to $\left|q_{\sigma} E_{0}\right| k /\left(m_{\sigma} \omega_{0}^{2}\right)$, as well as the dependence on polarisation of the pump wave, the direction of $\mathbf{k}$, and $\omega_{c \sigma} / \omega_{0}$, for the coupling between electrostatic Fourier-Laplace modes at different frequencies in the presence of the pump wave. $\beta_{\sigma}$ in 12 and (13) does not have any particular physical significance and may be changed by shifting point at which $t=0$. It is clear from $(12)$ and $(13)$ that only modes differing by integer multiples of $\omega_{0}$ interact. This is a manifestation of the frequency selection rule for parametric processes; in particular, the three-wave interactions are related to the $n= \pm 1$ terms. No coupling between modes with different $\mathbf{k}$ exists due to the dipole approximation, $\mathbf{k}_{0} \approx \mathbf{0}$. To recover the selection rules of (1) from the above equations, we use the fact that the requirement of real $\phi(\mathbf{r}, t)$ and $N_{\sigma}^{(1)}(\mathbf{x}, t)$ results in $\tilde{\phi}(\mathbf{k}, \omega)=\tilde{\phi}^{*}\left(-\mathbf{k},-\omega^{*}\right), \tilde{N}_{\sigma}^{(1)}(\mathbf{k}, \omega)=\tilde{N}_{\sigma}^{(1) *}\left(-\mathbf{k},-\omega^{*}\right)$ and $\chi_{\sigma}(\mathbf{k}, \omega)=\chi_{\sigma}^{*}\left(-\mathbf{k},-\omega^{*}\right)$, allowing the signs of $\mathbf{k}$ and $\operatorname{Re}(\omega)$ to be changed for any given mode. 
Specialising (12) and (13) to the case of an electron $(\sigma=e)$ and ion $(\sigma=i)$ plasma, when $\omega_{c i} / \omega_{0} \not \approx 1$ for any ionic species, we find $\mu_{i} / \mu_{e} \leq \mathrm{O}\left(m_{e} / m_{i}\right) \ll 1$ which allows us assume a linear ion response, $\mu_{i} \approx 0$, for moderate values of $\mu_{e}$. With this, $J_{n}\left( \pm \mu_{i}\right)=\delta_{n 0}$, and $(12)$ and $(13)$ become

$$
\begin{gathered}
\sum_{i} Z_{i} \tilde{N}_{i}^{(1)}(\mathbf{k}, \omega)=-\frac{\epsilon_{0} k^{2}}{e} \sum_{i} \chi_{i}(\mathbf{k}, \omega) \tilde{\phi}(\mathbf{k}, \omega), \\
\tilde{N}_{e}^{(1)}(\mathbf{k}, \omega)=\frac{\epsilon_{0} k^{2}}{e} \chi_{e}(\mathbf{k}, \omega) \sum_{n=-\infty}^{\infty} J_{n}\left(\mu_{e}\right) \mathrm{e}^{-\mathrm{i} n \beta_{e}} \tilde{\phi}\left(\mathbf{k}, \omega+n \omega_{0}\right), \\
\tilde{\phi}(\mathbf{k}, \omega)=\frac{e}{\epsilon_{0} k^{2}}\left[\sum_{i} Z_{i} \tilde{N}_{i}^{(1)}(\mathbf{k}, \omega)-\sum_{m=-\infty}^{\infty} J_{m}\left(-\mu_{e}\right) \mathrm{e}^{-\mathrm{i} m \beta_{e}} \tilde{N}_{e}^{(1)}\left(\mathbf{k}, \omega+m \omega_{0}\right)\right],
\end{gathered}
$$

where $e$ is the elementary charge, $Z_{i}=q_{i} / e$ is the charge number of the ionic species $i$, and all ionic terms of (12) have been summed. As the unknown quantities in the above equations are $\sum_{i} Z_{i} \tilde{N}_{i}^{(1)}\left(\mathbf{k}, \omega+n \omega_{0}\right), \tilde{N}_{e}^{(1)}\left(\mathbf{k}, \omega+n \omega_{0}\right)$ and $\tilde{\phi}\left(\mathbf{k}, \omega+n \omega_{0}\right)$, the addition of multiple ionic species does not complicate the problem with a linear ion response, provided that $\chi_{i, e}(\mathbf{k}, \omega)$ may still be determined. Substituting (17) into (15) and (16), and using Neumann's addition theorem to simplify the double sum in (16), $\sum_{n=-\infty}^{\infty} \sum_{m=-\infty}^{\infty} J_{n}\left(\mu_{e}\right) J_{m}\left(-\mu_{e}\right) \mathrm{e}^{-\mathrm{i}(n+m) \beta_{e}} \tilde{N}_{e}^{(1)}\left(\mathbf{k}, \omega+(n+m) \omega_{0}\right)=\tilde{N}_{e}^{(1)}(\mathbf{k}, \omega)$, we find

$$
\begin{aligned}
& {\left[1+\sum_{i} \chi_{i}(\mathbf{k}, \omega)\right] \sum_{i} Z_{i} \tilde{N}_{i}^{(1)}(\mathbf{k}, \omega)=\sum_{i} \chi_{i}(\mathbf{k}, \omega) \sum_{n=-\infty}^{\infty} J_{n}\left(-\mu_{e}\right) \mathrm{e}^{-\mathrm{i} n \beta_{e}} \tilde{N}_{e}^{(1)}\left(\mathbf{k}, \omega+n \omega_{0}\right),} \\
& {\left[1+\chi_{e}(\mathbf{k}, \omega)\right] \tilde{N}_{e}^{(1)}(\mathbf{k}, \omega)=\chi_{e}(\mathbf{k}, \omega) \sum_{n=-\infty}^{\infty} J_{n}\left(\mu_{e}\right) \mathrm{e}^{-\mathrm{i} n \beta_{e}} \sum_{i} Z_{i} \tilde{N}_{i}^{(1)}\left(\mathbf{k}, \omega+n \omega_{0}\right) .}
\end{aligned}
$$

These equations constitute the general basis for describing PDIs with electrostatic daughter waves in electron-ion plasmas within the dipole approximation when $\omega_{c i} \not \approx \omega_{0}$. Similar equations were derived by [4,11,40]; the expressions in [11] additionally contain terms related to plasma inhomogeneities, and the double sum in (11) of [40] vanishes by Neumann's addition theorem.

In the limit of weak coupling, $\mu_{e} \ll 1,(18)$ and $(19)$ may be solved by considering only the $(n=0, \pm 1)$-terms, giving a general parametric dispersion relation found in [4, 35, 40]; [4] only considers the $(n=0,-1)$-terms. However, since we are interested in a gyrotron pump wave, which satisfies $\omega_{0} \sim\left|\omega_{c e}\right| \gg \omega_{p i}, \omega_{c i}$, we can ignore the ion response at frequencies comparable to $\omega_{0}$, as was done by [10,11]. Associating $\omega$ with the lowfrequency daughter wave, we can thus write $\sum_{i} Z_{i} \tilde{N}_{i}^{(1)}\left(\mathbf{k}, \omega+n \omega_{0}\right) \approx \sum_{i} Z_{i} \tilde{N}_{i}^{(1)}(\mathbf{k}, \omega) \delta_{n 0}$, with which 19 becomes

$$
\tilde{N}_{e}^{(1)}\left(\mathbf{k}, \omega+n \omega_{0}\right)=\frac{\chi_{e}\left(\mathbf{k}, \omega+n \omega_{0}\right)}{1+\chi_{e}\left(\mathbf{k}, \omega+n \omega_{0}\right)} J_{-n}\left(\mu_{e}\right) \mathrm{e}^{\mathrm{i} n \beta_{e}} \sum_{i} Z_{i} \tilde{N}_{i}^{(1)}(\mathbf{k}, \omega) .
$$

Plugging this into (18), remembering that $J_{n}\left(-\mu_{e}\right) J_{-n}\left(\mu_{e}\right)=J_{n}^{2}\left(\mu_{e}\right)$, we arrive at a dispersion relation describing PDIs for pump waves of arbitrary amplitude in the EC 
frequency range,

$$
1+\sum_{i} \chi_{i}(\mathbf{k}, \omega)=\sum_{i} \chi_{i}(\mathbf{k}, \omega) \sum_{n=-\infty}^{\infty} J_{n}^{2}\left(\mu_{e}\right) \frac{\chi_{e}\left(\mathbf{k}, \omega+n \omega_{0}\right)}{1+\chi_{e}\left(\mathbf{k}, \omega+n \omega_{0}\right)},
$$

also found in [10]. In the weak coupling limit, generally relevant to gyrotron radiation (which has power levels $\lesssim 1 \mathrm{MW}$ ) [42], the Bessel functions may be Taylor expanded to order $\mu_{e}^{2}\left(J_{0}^{2}\left(\mu_{e}\right) \approx 1-\mu_{e}^{2} / 2, J_{ \pm 1}^{2}\left(\mu_{e}\right) \approx \mu_{e}^{2} / 4\right.$, the remaining terms are negligible), yielding

$1+\sum_{i} \chi_{i}(\mathbf{k}, \omega)+\chi_{e}(\mathbf{k}, \omega)=\frac{\mu_{e}^{2}}{4} \sum_{i} \chi_{i}(\mathbf{k}, \omega)\left[2-\frac{1+\chi_{e}(\mathbf{k}, \omega)}{1+\chi_{e}\left(\mathbf{k}, \omega-\omega_{0}\right)}-\frac{1+\chi_{e}(\mathbf{k}, \omega)}{1+\chi_{e}\left(\mathbf{k}, \omega+\omega_{0}\right)}\right]$

the identity $-\chi_{e}(\mathbf{k}, \omega) /\left[1+\chi_{e}(\mathbf{k}, \omega)\right]+\chi_{e}\left(\mathbf{k}, \omega \pm \omega_{0}\right) /\left[1+\chi_{e}\left(\mathbf{k}, \omega \pm \omega_{0}\right)\right]=1 /\left[1+\chi_{e}(\mathbf{k}, \omega)\right]-$ $1 /\left[1+\chi_{e}\left(\mathbf{k}, \omega \pm \omega_{0}\right)\right]$ has been used to rewrite the terms in the bracket on the right hand side. Introducing the dielectric function $\epsilon(\mathbf{k}, \omega)=1+\sum_{i} \chi_{i}(\mathbf{k}, \omega)+\chi_{e}(\mathbf{k}, \omega)$, noting that $\epsilon\left(\mathbf{k}, \omega \pm \omega_{0}\right) \approx 1+\chi_{e}\left(\mathbf{k}, \omega \pm \omega_{0}\right)$, and neglecting the first term in the bracket on the right hand side of (22), we arrive at the weak coupling parametric dispersion relation,

$\epsilon(\mathbf{k}, \omega)=-\frac{\mu_{e}^{2}}{4} \sum_{i} \chi_{i}(\mathbf{k}, \omega)\left[1+\chi_{e}(\mathbf{k}, \omega)\right]\left[\frac{1}{\epsilon\left(\mathbf{k}, \omega-\omega_{0}\right)}+\frac{1}{\epsilon\left(\mathbf{k}, \omega+\omega_{0}\right)}\right]$.

This form illustrates the coupling between the low-frequency daughter wave, characterised by $\left(\mathbf{k}, \omega=\omega_{1}+\mathrm{i} \gamma\right)$, the down-shifted high-frequency daughter wave, characterised by $\left(\mathbf{k}, \omega-\omega_{0}=-\omega_{2}+\mathrm{i} \gamma\right)$, and the up-shifted high-frequency daughter wave, characterised by $\left(\mathbf{k}, \omega+\omega_{0}=\omega_{3}+\mathrm{i} \gamma\right)$, due to the finite amplitude of the pump wave (through $\mu_{e}^{2}$ ). The coupling will be extremely small, and the low-frequency daughter wave response almost linear $(\epsilon(\mathbf{k}, \omega) \approx 0)$, for the usual case of $\mu_{e} \ll 1$, unless $\epsilon\left(\mathbf{k}, \omega \pm \omega_{0}\right) \approx 0$. Thus, the high-frequency modes should correspond (almost) to linear plasma modes, which also justifies neglecting the first term in the bracket on the right hand side of $(22)$. If $\epsilon(\mathbf{k}, \omega) \approx 0$ remains valid, a PDI ( $\gamma>0$ in a homogeneous plasma) may occur for $\mu_{e} \ll 1$, confirming the correctness of the simplified picture in figure 2 .

Parametric dispersion relations equivalent to (23) are found in 20, 40]; note that the one given by [40 is derived assuming an LH pump wave, and thus based on some rather different assumptions. The parametric dispersion relation from [19] agrees with (23) within the dipole approximation. The general weak coupling parametric dispersion relation in (28a) of [35] agrees with our (23) for $\chi_{i}\left(\mathbf{k}, \omega \pm \omega_{0}\right) \approx 0$ and $\chi_{e}\left(\mathbf{k}, \omega \pm \omega_{0}\right) \approx-1$, as we have assumed. However, in going from (28a) to (28b), 35] assumes $\left|\chi_{e, i}(\mathbf{k}, \omega)\right| \gg\left|\chi_{e, i}\left(\mathbf{k}, \omega \pm \omega_{0}\right)\right|$, which causes $1+\chi_{e}(\mathbf{k}, \omega) \rightarrow \chi_{e}(\mathbf{k}, \omega)$ in its version of (23), generally reducing the validity of the subsequent formulae to PDIs with low-frequency daughter waves satisfying the quasi-neutrality condition $\left|\chi_{e}(\mathbf{k}, \omega)\right| \gg 1$; this is particularly not satisfied by warm LH wave propagating nearly perpendicularly to $\mathbf{B}$, which is the case of interest in the present paper. The parametric dispersion relation from [4] also agrees with (23) for $\chi_{i}\left(\mathbf{k}, \omega \pm \omega_{0}\right) \approx 0$ and $\chi_{e}\left(\mathbf{k}, \omega \pm \omega_{0}\right) \approx-1$ if only the down-shifted high-frequency daughter wave is considered. Ott, Hui and Chu [18] 
derive their parametric dispersion relation from rather different arguments about the ponderomotive force acting on the electrons and the related density perturbations experienced by the ions. The final result does, however, resemble (23) if the substitutions $1+\chi_{e}(\mathbf{k}, \omega) \rightarrow \chi_{e}(\mathbf{k}, \omega)$ and $\sum_{i} \chi_{i}(\mathbf{k}, \omega) \rightarrow 1+\sum_{i} \chi_{i}(\mathbf{k}, \omega)$ are made; the variational approach of [5] appears to produce the same result in the weak coupling and dipole limit, as shown in Subsection 3.2. The results of [5, 18] do not agree with the previous ones, and thus a re-examination of these and related expressions, e.g. the one in [43], may be of interest. We note that all expressions agree for low-frequency daughter waves satisfying the quasi-neutrality conditions $\left|\chi_{i, e}(\mathbf{k}, \omega)\right| \gg 1$, but that (23) is preferable for pump waves in the EC frequency range where this condition may not be satisfied.

(23) does still not give $\gamma$ explicitly and must in general be solved numerically. However, by using the earlier results we can obtain relatively simple expressions for the limiting cases of most interest. We consider the down-shifted high-frequency mode to be on resonance, $\epsilon\left(\mathbf{k},-\omega_{2}+\mathrm{i} \gamma\right) \approx 0$, and neglect the contribution of the up-shifted highfrequency mode, which is permissible if the damping rates of the high-frequency modes are small compared to $\omega_{1}$. In this case we can take $\operatorname{Re}\left[\epsilon\left(\mathbf{k},-\omega_{2}\right)\right]=0$ and expand $\epsilon\left(\mathbf{k},-\omega_{2}+\mathrm{i} \gamma\right)$ around $\left(\mathbf{k},-\omega_{2}\right)$, using $\epsilon\left(\mathbf{k},-\omega_{2}\right)=\epsilon^{*}\left(-\mathbf{k}, \omega_{2}\right)$, to find $\epsilon\left(\mathbf{k}, \omega-\omega_{0}\right) \approx$ $-\left.\mathrm{i}\{\partial \operatorname{Re}[\epsilon(-\mathbf{k}, \omega)] / \partial \omega\}\right|_{\omega=\omega_{2}}\left[\gamma+\Gamma\left(-\mathbf{k}, \omega_{2}\right)\right]$, where the linear damping rate of the downshifted high-frequency mode is $\Gamma\left(-\mathbf{k}, \omega_{2}\right)=\operatorname{Im}\left[\epsilon\left(-\mathbf{k}, \omega_{2}\right)\right] /\left.\{\partial \operatorname{Re}[\epsilon(-\mathbf{k}, \omega)] / \partial \omega\}\right|_{\omega=\omega_{2}}$. Plugging the above results into (23), and isolating $\gamma+\Gamma\left(-\mathbf{k}, \omega_{2}\right)$, gives

$$
\begin{aligned}
\gamma+\Gamma\left(-\mathbf{k}, \omega_{2}\right) & =-\frac{\mu_{e}^{2}}{4} \operatorname{Re}\left\{\frac{\mathrm{i} \sum_{i} \chi_{i}(\mathbf{k}, \omega)\left[1+\chi_{e}(\mathbf{k}, \omega)\right]}{\left.\epsilon(\mathbf{k}, \omega)\{\partial \operatorname{Re}[\epsilon(-\mathbf{k}, \omega)] / \partial \omega\}\right|_{\omega=\omega_{2}}}\right\} \\
& =\frac{\mu_{e}^{2}}{4} \frac{\left|\sum_{i} \chi_{i}(\mathbf{k}, \omega)\right|^{2} \operatorname{Im}\left[\chi_{e}(\mathbf{k}, \omega)\right]+\left|1+\chi_{e}(\mathbf{k}, \omega)\right|^{2} \sum_{i} \operatorname{Im}\left[\chi_{i}(\mathbf{k}, \omega)\right]}{\left.|\epsilon(\mathbf{k}, \omega)|^{2}\{\partial \operatorname{Re}[\epsilon(-\mathbf{k}, \omega)] / \partial \omega\}\right|_{\omega=\omega_{2}}} .
\end{aligned}
$$

The above equations lead to two different expressions for $\gamma$ depending on the conditions satisfied by the low-frequency mode. The two cases are known as resonant and nonresonant PDIs, respectively. For resonant PDIs, the low-frequency mode is also weakly damped, $\omega_{1} \gg \Gamma\left(\mathbf{k}, \omega_{1}\right)$, and we can take $\operatorname{Re}\left[\epsilon\left(\mathbf{k}, \omega_{1}\right)\right]=0$ and expand $\epsilon\left(\mathbf{k}, \omega_{1}+\mathrm{i} \gamma\right)$ around $\left(\mathbf{k}, \omega_{1}\right)$ to find $\left.\epsilon(\mathbf{k}, \omega) \approx \mathrm{i}\{\partial \operatorname{Re}[\epsilon(\mathbf{k}, \omega)] / \partial \omega\}\right|_{\omega=\omega_{1}}\left[\gamma+\Gamma\left(\mathbf{k}, \omega_{1}\right)\right]$, with which 24 may be rewritten as

$$
\left[\gamma+\Gamma\left(\mathbf{k}, \omega_{1}\right)\right]\left[\gamma+\Gamma\left(-\mathbf{k}, \omega_{2}\right)\right]=-\frac{\mu_{e}^{2}}{4} \frac{\sum_{i} \operatorname{Re}\left[\chi_{i}\left(\mathbf{k}, \omega_{1}\right)\right]\left\{1+\operatorname{Re}\left[\chi_{e}\left(\mathbf{k}, \omega_{1}\right)\right]\right\}}{\left.\left.\{\partial \operatorname{Re}[\epsilon(\mathbf{k}, \omega)] / \partial \omega\}\right|_{\omega=\omega_{1}}\{\partial \operatorname{Re}[\epsilon(-\mathbf{k}, \omega)] / \partial \omega\}\right|_{\omega=\omega_{2}}},
$$

where the order 0 expressions, $\chi_{e}(\mathbf{k}, \omega) \approx \operatorname{Re}\left[\chi_{e}\left(\mathbf{k}, \omega_{1}\right)\right]$ and $\chi_{i}(\mathbf{k}, \omega) \approx \operatorname{Re}\left[\chi_{i}\left(\mathbf{k}, \omega_{1}\right)\right]$, have been used in the numerator; (25) is similar to (A2) from [4] for $\chi_{i}\left(-\mathbf{k}, \omega_{2}\right) \approx 0$ and $\chi_{e}\left(-\mathbf{k}, \omega_{2}\right) \approx-1$. This quadratic equation for $\gamma$ is easily solved (showing only the possibly positive root),

$$
\begin{array}{r}
\gamma=\frac{1}{2}\left[\sqrt{\left[\Gamma\left(\mathbf{k}, \omega_{1}\right)-\Gamma\left(-\mathbf{k}, \omega_{2}\right)\right]^{2}-\frac{\mu_{e}^{2} \sum_{i} \operatorname{Re}\left[\chi_{i}\left(\mathbf{k}, \omega_{1}\right)\right]\left\{1+\operatorname{Re}\left[\chi_{e}\left(\mathbf{k}, \omega_{1}\right)\right]\right\}}{\left.\left.\{\partial \operatorname{Re}[\epsilon(\mathbf{k}, \omega)] / \partial \omega\}\right|_{\omega=\omega_{1}}\{\partial \operatorname{Re}[\epsilon(-\mathbf{k}, \omega)] / \partial \omega\}\right|_{\omega=\omega_{2}}}}\right. \\
\left.-\Gamma\left(\mathbf{k}, \omega_{1}\right)-\Gamma\left(-\mathbf{k}, \omega_{2}\right)\right],(26)
\end{array}
$$


and the PDI threshold $(\gamma>0)$ in a homogeneous plasma may be expressed as the condition

$$
\mu_{e}^{2}>-\frac{4 \operatorname{Im}\left[\epsilon\left(\mathbf{k}, \omega_{1}\right)\right] \operatorname{Im}\left[\epsilon\left(-\mathbf{k}, \omega_{2}\right)\right]}{\sum_{i} \operatorname{Re}\left[\chi_{i}\left(\mathbf{k}, \omega_{1}\right)\right]\left\{1+\operatorname{Re}\left[\chi_{e}\left(\mathbf{k}, \omega_{1}\right)\right]\right\}} .
$$

For non-resonant PDIs, the low-frequency mode is not weakly damped $\left(\epsilon\left(\mathbf{k}, \omega_{1}\right) \not \approx 0\right)$, and the right hand side of (24) may be approximated by its value at $\omega=\omega_{1}$ without the assumption of small $\operatorname{Im}\left[\chi_{e}(\mathbf{k}, \omega)\right]$ and $\operatorname{Im}\left[\chi_{i}(\mathbf{k}, \omega)\right]$,

$\gamma=\frac{\mu_{e}^{2}}{4} \frac{\left|\sum_{i} \chi_{i}\left(\mathbf{k}, \omega_{1}\right)\right|^{2} \operatorname{Im}\left[\chi_{e}\left(\mathbf{k}, \omega_{1}\right)\right]+\left|1+\chi_{e}\left(\mathbf{k}, \omega_{1}\right)\right|^{2} \sum_{i} \operatorname{Im}\left[\chi_{i}\left(\mathbf{k}, \omega_{1}\right)\right]}{\left.\left|\epsilon\left(\mathbf{k}, \omega_{1}\right)\right|^{2}\{\partial \operatorname{Re}[\epsilon(-\mathbf{k}, \omega)] / \partial \omega\}\right|_{\omega=\omega_{2}}}-\Gamma\left(-\mathbf{k}, \omega_{2}\right) ;$

an equation similar to 28 is used as a starting point by [2. 28) yields the homogeneous plasma PDI threshold,

$$
\mu_{e}^{2}>\frac{4 \operatorname{Im}\left[\epsilon\left(-\mathbf{k}, \omega_{2}\right)\right]\left|\epsilon\left(\mathbf{k}, \omega_{1}\right)\right|^{2}}{\left|\sum_{i} \chi_{i}\left(\mathbf{k}, \omega_{1}\right)\right|^{2} \operatorname{Im}\left[\chi_{e}\left(\mathbf{k}, \omega_{1}\right)\right]+\left|1+\chi_{e}\left(\mathbf{k}, \omega_{1}\right)\right|^{2} \sum_{i} \operatorname{Im}\left[\chi_{i}\left(\mathbf{k}, \omega_{1}\right)\right]} .
$$

It is seen that both the resonant and the non-resonant PDIs in a homogeneous plasma need the pump wave amplitude $\left(\left|E_{0}\right| \propto \mu_{e}\right)$ to exceed a certain threshold, principally determined by the daughter wave damping, in order to occur. However, while the resonant PDI threshold increases with both the low- and high-frequency daughter wave damping, through $\operatorname{Im}\left[\epsilon\left(\mathbf{k}, \omega_{1}\right)\right]$ and $\operatorname{Im}\left[\epsilon\left(-\mathbf{k}, \omega_{2}\right)\right]$, the non-resonant PDI threshold increases with damping of the high-frequency daughter wave, but does not occur without damping of the low-frequency daughter wave. The above observations point to an important difference between the resonant and non-resonant PDIs. The resonant PDI can be seen as a pure wave-wave interaction, in which the beating of one daughter wave with the pump wave amplifies the other daughter wave and vice versa [44]. This results in a fairly symmetric treatment of the two daughter waves, which is generally adequately described by fluid models. The non-resonant PDI can rather be seen as a wave-particle interaction, in which energy is transferred from the pump wave to the high-frequency daughter wave through nonlinear Landau damping of the low-frequency daughter wave [45]. This results in a treatment of the high-frequency daughter wave which is virtually independent of the low-frequency daughter wave and generally requires a kinetic description.

\subsection{PDIs in Inhomogeneous Plasmas}

Although we have now derived thresholds for PDIs in homogeneous plasmas, these are generally far below the thresholds observed in real inhomogeneous plasmas. The main reason for this is that the $\mathbf{k}$ selection rule for the waves involved in a PDI is only approximately satisfied in a small region through which a significant amplification of the thermal background daughter waves has to occur in order for the PDI to have measurable consequences. Within the geometric optics approximation, we may convert the temporal growth rate in a homogeneous medium into a local spatial growth rate $\gamma / v_{g}(\mathbf{k}, \omega)$ along the ray, where $v_{g}(\mathbf{k}, \omega)=|\{\partial \operatorname{Re}[\epsilon(\mathbf{k}, \omega)] / \partial \mathbf{k}\} /\{\partial \operatorname{Re}[\epsilon(\mathbf{k}, \omega)] / \partial \omega\}|$ is the magnitude of the group velocity (the speed of energy transport along the ray). From 
this, the power gain of the high-frequency daughter waves in the case of non-resonant PDIs, $G=\ln \left(P_{\text {out }} / P_{\text {in }}\right)$, may be calculated as [18,46],

$$
G=\int_{\text {ray }} \frac{2 \gamma\left(\mathbf{r}(s), \mathbf{k}(s), \omega_{1}, \omega_{2}\right)}{v_{g}\left(\mathbf{r}(s),-\mathbf{k}(s), \omega_{2}\right)} \mathrm{d} s
$$

where the integral is over a segment of the ray of the high-frequency daughter wave for given initial conditions and $s$ is a parameter characterising length along the ray. Assuming that $\gamma$ is only significant in a small region around the point $s=s_{r}$, at which the selection rule is exactly satisfied for a particular low-frequency quasi-mode, and that the ray only traverses this region once, we can write

$$
G \approx \frac{2 \gamma\left(\mathbf{r}\left(s_{r}\right), \mathbf{k}\left(s_{r}\right), \omega_{1}, \omega_{2}\right) \ell\left(s_{r}\right)}{v_{g}\left(\mathbf{r}\left(s_{r}\right),-\mathbf{k}\left(s_{r}\right), \omega_{2}\right)},
$$

with $\ell\left(s_{r}\right)$ being the length along the ray around $s_{r}$ with significant $\gamma$. A threshold is obtained by requiring $G$ to be sufficiently large. For instance, [13] defines the threshold to be $G>2 \pi$, corresponding to $P_{\text {out }} / P_{\text {in }}>535$, which we shall also use, keeping in mind that PDIs may be observable for slightly lower values of $G$. Note that the threshold may be significantly reduced if the amplification region is traversed several times by the ray of the high-frequency daughter wave without significant damping between the individual passes. This may happen if the high-frequency daughter wave is trapped due to a non-monotonic plasma profile around the amplification region [16] or in the case of a backscattering instability [17]. However, $\gamma>0$ represents the lowest possible threshold in all cases.

For the case of a resonant PDI in a plasma slab with monotonically varying parameters along the $x$-direction and daughter waves propagating along the $x$-direction, [12 14] showed that, within the WKB approximation,

$$
G=\frac{2 \pi \gamma^{2}\left(x_{r}, k_{x}\left(x_{r}\right), \omega_{1}, \omega_{2}\right) l^{2}\left(x_{r}, \omega_{1}, \omega_{2}\right)}{v_{g}\left(x_{r}, k_{x}\left(x_{r}\right), \omega_{1}\right) v_{g}\left(x_{r},-k_{x}\left(x_{r}\right), \omega_{2}\right)} .
$$

Here $x=x_{r}$ is the $x$-value at which the selection rules are exactly satisfied, $l^{2}\left(x_{r}, \omega_{1}, \omega_{2}\right)=1 /\left|\left\{\left.\left[\partial k_{x}\left(x, \omega_{1}\right) / \partial x\right]\right|_{x=x_{r}}-\left.\left[\partial k_{x}\left(x, \omega_{2}\right) / \partial x\right]\right|_{x=x_{r}}\right\}\right|$ is the length scale over which the $k_{x}(x)$-selection rule is well satisfied, and we have retained the dipole approximation, $k_{0}(x) \approx 0$. This $G$ is seen to be quite similar to the one for nonresonant decay from (31); the main difference is that the high-frequency and lowfrequency daughter waves enter $G$ in a symmetric fashion, as expected for the resonant PDI, and that $l\left(x_{r}\right)$, unlike $\ell\left(s_{r}\right)$, is not arbitrary. While the application of 32 to a realistic $3 \mathrm{D}$ plasma may not provide the actual threshold, since it only addresses wave propagation along a gradient with a constant direction, it should still provide an order of magnitude estimate of the resonant PDI threshold in a non-monotonic plasma. For the non-resonant PDI, a similar estimate may be obtained by only considering propagation along the $x$-direction and setting $\ell\left(s_{r}\right) \approx l\left(x_{r}, \omega_{1}, \omega_{2}\right)$, such that (31) becomes $G \approx 2 \gamma\left(x_{r}, k\left(x_{r}\right), \omega_{1}, \omega_{2}\right) l\left(x_{r}, \omega_{1}, \omega_{2}\right) / v_{g}\left(x_{r},-k_{x}\left(x_{r}\right), \omega_{2}\right)$. 


\section{PDIs near the UHR}

So far, PDIs involving gyrotron radiation with $\omega_{0} \sim\left|\omega_{c e}\right|$ have been discussed in a rather general fashion; we now specialise to the case of PDIs near the UHR. As is known from the theory of cold electromagnetic plasma waves, $\mathrm{X}$-mode radiation has a principal resonance $\left(k_{0} \rightarrow \infty\right.$, or $v_{g}\left(\mathbf{k}_{0}, \omega_{0}\right) \rightarrow 0$, for $\left.\mathbf{k}_{0} \perp \mathbf{B}\right)$ at the UH frequency, $\omega_{U H}^{2}=\omega_{c e}^{2}+\omega_{p e}^{2}$, with a propagating X-mode only existing on the side where $\omega_{0}<\omega_{U H}$. Under normal circumstances, X-mode radiation encountering the UHR is converted linearly into an electron Bernstein/warm UH wave [38], the dispersion relation of which we derive below. However, PDIs are also likely to occur near the UHR because $\left|E_{0}\right|$ (and hence $\mu_{e}$ ) of the X-mode radiation becomes significantly enhanced compared with its normal value for gyrotron radiation, due to a low group velocity and full-wave effects [15] combined with the fact that linear Landau and cyclotron damping remains small, unless $\omega_{U H} \approx n\left|\omega_{c e}\right|$ for $n \in \mathbb{N}[38]$. In this paper we focus on direct parametric decay of the $\mathrm{X}$-mode radiation into electrostatic daughter waves before the linear mode conversion to warm UH waves takes place as was done by [1 5$]$; this requires propagating daughter waves to exist in the region where $\omega_{0}<\omega_{U H}$. For PDIs of the linearly converted warm UH waves see [17,21,22].

This work is, as previously mentioned, primarily motivated by the observation of strong anomalous scattering during some CTS experiments at the ASDEX Upgrade tokamak [6]; observations resembling the ones from ASDEX Upgrade have also been made during CTS experiments at the LHD stellarator [7, 8]. The CTS spectral power density obtained in one of these experiments (ASDEX Upgrade discharge 28286) is seen in figure 3. The figure shows peaks with frequency shifts of approximately $850 \mathrm{MHz}$ relative to the gyrotron frequency $\left(\omega_{0} /(2 \pi)=104.93 \mathrm{GHz}\right)$ developing at $t=2.100 \mathrm{~s}$ and becoming well separated from the strong signal at small frequency shifts for $t \geq 2.500 \mathrm{~s}$. The frequency shift slightly exceeds the LH frequency of approximately $700 \mathrm{MHz}$ at the UHR of the gyrotron radiation in the experiment (for reference, the plasma parameters are $N_{e}^{(0)} \approx 2.8 \times 10^{19} \mathrm{~m}^{-3}, T_{e} \approx 300 \mathrm{eV}$ and $B \approx 3.35 \mathrm{~T}$ at this location), and the occurrence of the peaks is additionally strongly correlated with a significant amount of power reaching the UHR in X-mode. The latter point is illustrated in figure 4 (the details of which are discussed below) through traces of the CTS probe rays, done using a code based on analytical approximations to the relativistic dispersion relation developed in [47, 48], at various times in the discharge: virtually no X-mode radiation reaches the UHR in the main plasma at the early time point, $t=1.300 \mathrm{~s}$, while a smaller fraction of the X-mode beam may reach it at the intermediate time point, $t=2.100 \mathrm{~s}$, and a significant fraction of the X-mode beam will reach it at the late time points, $t \geq 2.500 \mathrm{~s}$. Note, however, that the traced CTS probe ray does not reach the UHR in the main plasma before $t=2.900 \mathrm{~s}$.

As seen in figure 4, the UHR is only accessible to X-mode radiation launched from its high-field side in tokamak plasmas due to the location of the $\mathrm{R}$ cut-off. For CTS applications, access to the entire plasma volume is desired, so the $105 \mathrm{GHz}$ gyrotron 

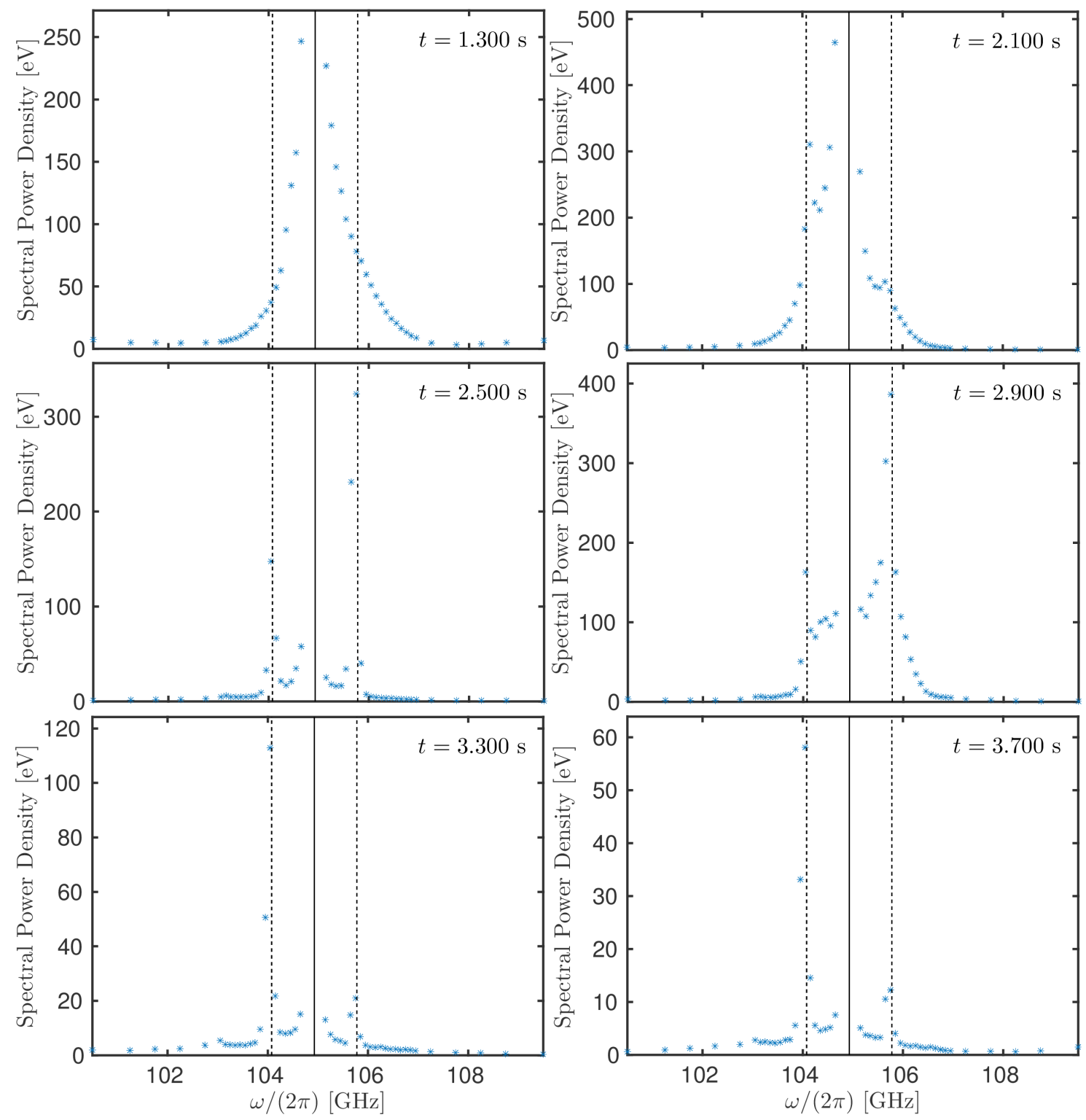

Figure 3. CTS spectral power density (in $\mathrm{eV}$, as is customary [6]) around the gyrotron frequency $\omega_{0} /(2 \pi)=104.93 \mathrm{GHz}$, marked by the full line, in ASDEX Upgrade discharge 28286. At the early time point, $t=1.300 \mathrm{~s}$, no peaks are observed; at the intermediate time point, $t=2.100 \mathrm{~s}$, peaks with frequency shifts of approximately $850 \mathrm{MHz}$, marked by dashed lines, start developing; at late time points, $t \geq 2.500 \mathrm{~s}$, these peaks become well-separated from the signal at small frequency shifts.

radiation is launched in O-mode from the low-field side. Thus, in order for power to reach the UHR, a reflection of the injected O-mode radiation from the high-field side wall, in which the reflected radiation is at least partially converted to X-mode, is necessary; a similar mechanism was invoked by [32] to explain generation of fast ions during 1st harmonic O-mode ECRH, with a low-field side launch, at the TCA tokamak.

To understand the trajectories of the rays in figure 4, as well as the characteristics of the PDIs later on, we plot the development of the $N_{e}^{(0)}$ - and $T_{e}$-profiles obtained from 

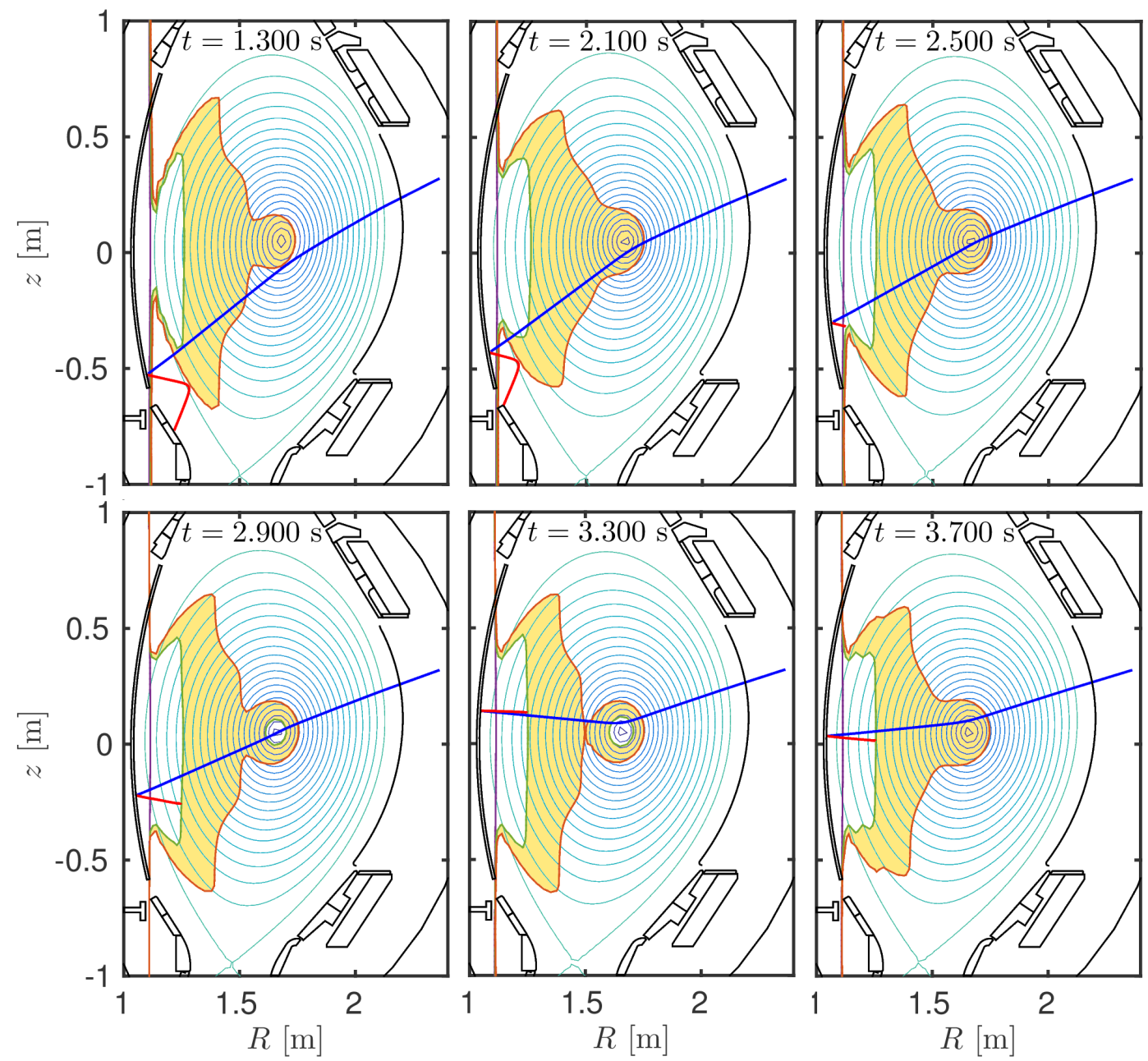

Figure 4. Traces of the central rays of the $105 \mathrm{GHz}$ O-mode radiation injected from the low-field side (blue line) and the $105 \mathrm{GHz} \mathrm{X}$-mode radiation reflected from the high-field side vessel wall (red line), projected into the $(R, z)$-plane of the cylindrical tokamak coordinate system, in ASDEX Upgrade discharge 28286 at $t=\{1.300 \mathrm{~s}, 2.100 \mathrm{~s}, 2.500 \mathrm{~s}, 2.900 \mathrm{~s}, 3.300 \mathrm{~s}, 3.700 \mathrm{~s}\}$. The green, purple, and orange lines indicate the locations of the UHR, the ECR, and the R cut-off, respectively; no propagating X-mode exists in the shaded areas; the background contours are closed magnetic flux surfaces indicating the position of the main plasma. At $t=1.300 \mathrm{~s}$, virtually none of the reflected X-mode radiation reaches the UHR, while part of the reflected X-mode radiation may reach it at $t=2.100 \mathrm{~s}$. For $t \geq 2.500 \mathrm{~s}$, most of the reflected X-mode radiation reaches the UHR, although the traced central beam ray does not reach it in the main plasma before $t=2.900 \mathrm{~s}$. These observations are consistent with the $850 \mathrm{MHz}$ shifted peaks in figure 3 being caused by a PDI occurring at the UHR. Rays have been traced using a code based on analytical approximations to the relativistic dispersion relation developed in 4748 . The $N_{e}^{(0)}$ - and $T_{e}$-profiles have been generated using IDA [49] and the magnetic ASDEX Upgrade equilibria obtained using the CLISTE code 50 . 

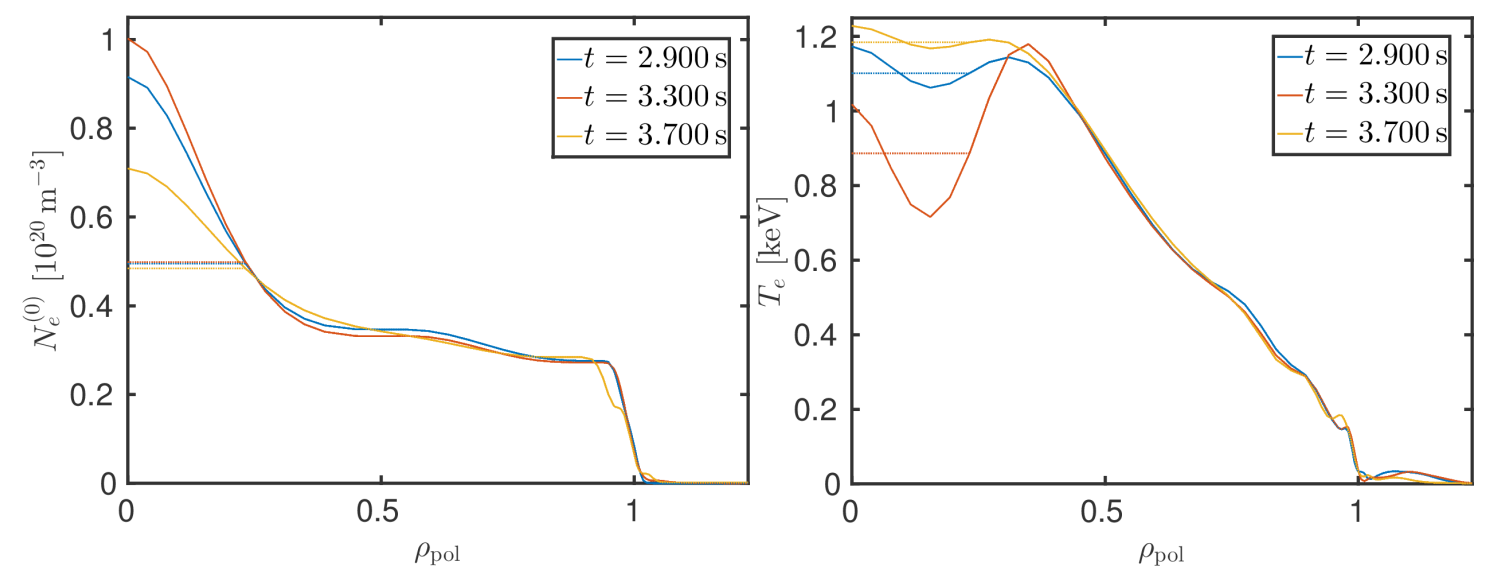

Figure 5. Development of $N_{e}^{(0)}$ and $T_{e}$ versus the normalised poloidal flux coordinate, $\rho_{\text {pol }}$, in ASDEX Upgrade discharge 28286. The IDA profiles are marked by full lines, while the dotted lines represent the flat central profiles used to estimate the uncertainty resulting from the poorly diagnosed plasma centre.

integrated data analysis (IDA) [49] versus the normalised poloidal flux coordinate, $\rho_{\text {pol }}$ $\left(\rho_{\text {pol }}=0\right.$ at the plasma centre and $\rho_{\text {pol }}=1$ at the last closed flux surface, magnetic equilibria are calculated using the CLISTE code [50]), for $t=\{2.900 \mathrm{~s}, 3.300 \mathrm{~s}, 3.700 \mathrm{~s}\}$ in figure 5. Evidently, $N_{e}^{(0)}$ is peaked around the plasma centre, resulting in the large refraction taking place here. However, the central peaking of $N_{e}^{(0)}$ is an extrapolation, as no measurements of the plasma parameters for $\rho_{\text {pol }}<0.2$ were possible in the discharge. In order to address this issue, rays have also been traced for flat central $N_{e}^{(0)}$ and $T_{e}$ profiles, marked by dotted lines in figure 5. These rays differ from the ones shown in figure 4 by suffering virtually no refraction at the plasma centre, but the PDI relevant conclusions, and the plasma parameters at the UHR (encountered at $\rho_{\text {pol }} \approx 0.8$ ), remain the same; the only difference is that the CTS probe ray encounters the UHR in the main plasma for $t \geq 2.500 \mathrm{~s}$ with the flat profiles. To have a rough estimate of the experimental uncertainty of the PDI threshold and frequency shift, all subsequent calculations are carried out for both the peaked and the flat profiles shown in figure 5; analyses are only performed for $t \geq 2.900 \mathrm{~s}$.

For PDIs to occur near the UHR by the mechanism described above, a nonnegligible fraction of the reflected power, $\mathcal{F}$, should be coupled into the main plasma in X-mode and, additionally, absorption at the EC resonance (ECR) should not be excessive. These points are also addressed by the ray traces, since the evolution of the wave polarisation along the $\mathrm{O}$-mode and $\mathrm{X}$-mode rays, as well as during the high-field side wall reflection, determines $\mathcal{F}$, while the total optical thickness of the O-mode and $\mathrm{X}$-mode rays, $\tau=-G$, determines the fraction of power transmitted through the ECR, $\mathrm{e}^{-\tau}$. The total power reaching the UHR is approximately $P=\mathcal{F} \mathrm{e}^{-\tau} P_{0}$, with $P_{0}$ being the gyrotron power. Figure 6 shows $\mathcal{F}, \mathrm{e}^{-\tau}$ and $\mathcal{F} \mathrm{e}^{-\tau}$ for the CTS probe rays reaching the UHR in the main plasma $(t \geq 2.900 \mathrm{~s})$. $\mathcal{F}$ is calculated as the square norm of the projection of the reflected ray polarisation vector along the X-mode polarisation vector 


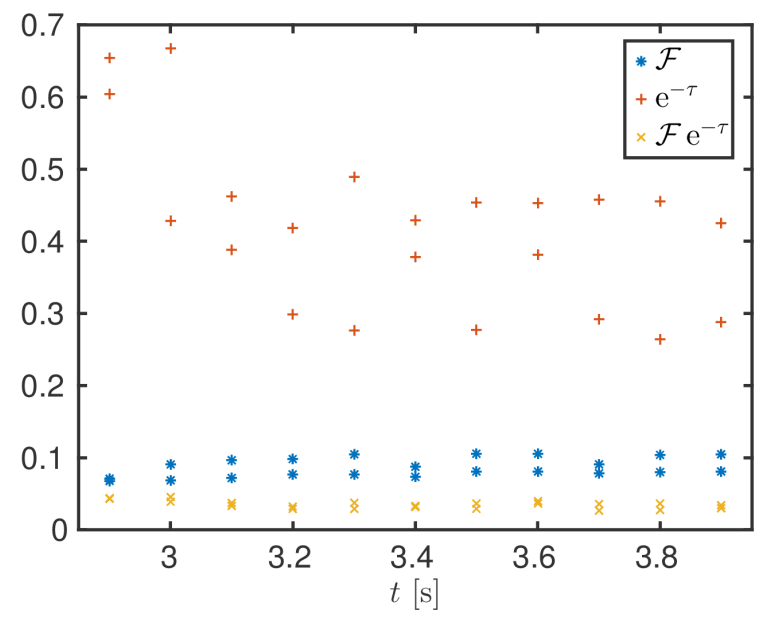

Figure 6. Fraction of reflected power coupled to the plasma in X-mode, $\mathcal{F}$, fraction of power transmitted by the plasma, $\mathrm{e}^{-\tau}$, and fraction of power reaching the UHR in $\mathrm{X}$-mode, $\mathcal{F} \mathrm{e}^{-\tau}$, versus $t$ in ASDEX Upgrade discharge 28286.

at the ECR or the last closed flux surface, depending on which of these is encountered first; the high-field side wall is treated as a locally plane, perfectly reflecting mirror. As seen in figure $6, \mathcal{F} \approx 0.1$ in all cases where the CTS probe ray reaches the UHR in the main plasma. $\tau$ is calculated using a relativistic model and is found to have moderate values, $\min \left(\mathrm{e}^{-\tau}\right)=0.265$, meaning that absorption at the ECR is non-excessive in all cases. Accounting for both $\tau$ and $\mathcal{F}$, the amount of gyrotron power reaching the UHR in $\mathrm{X}$-mode is $P=\mathcal{F} \mathrm{e}^{-\tau} P_{0} \approx 0.04 P_{0}$, corresponding to $P \approx 8 \mathrm{~kW}$ for the nominal gyrotron power, $P_{0}=200 \mathrm{~kW}$, in the experiment. This power level is of an order of magnitude where PDIs may be expected to occur near the UHR in a tokamak [3], allowing them to account for the CTS spectrum in figure 3. We note that both $\mathcal{F}$ and $\mathrm{e}^{-\tau}$ are quite sensitive functions of the toroidal gyrotron injection angle in the ASDEX Upgrade equilibria considered: when the gyrotron radiation is injected poloidally, virtually none of the reflected radiation is coupled to the plasma in X-mode, $\mathcal{F} \approx 0$, but the ECR is optically thin, $\mathrm{e}^{-\tau} \sim 1$; as the toroidal gyrotron injection angle increases, $\mathcal{F}$ grows while $\mathrm{e}^{-\tau}$ decays, and when the numerical value of the toroidal gyrotron injection angle exceeds approximately $10^{\circ}$, the ECR becomes optically thick, $\mathrm{e}^{-\tau} \approx 0$. The combination of these effects results in $\max \left(\mathcal{F} \mathrm{e}^{-\tau}\right) \approx 0.04$ which coincides with the toroidal gyrotron injection angle of approximately $-5^{\circ}$ used throughout the discharge. The above observations provide simple experimental prescriptions for suppressing PDIs at the UHR in 105 GHz O-mode CTS experiments at ASDEX Upgrade. However, it is noted that PDI suppression by a completely poloidal injection requires both the width of the gyrotron beam and its angular divergence to be very small, while PDI suppression by a large toroidal injection angle requires $B$ to be large enough for the ECR to occur between the high-field side wall and the UHR, ideally inside the last closed flux surface; the latter prescription has successfully suppressed PDIs in $105 \mathrm{GHz}$ O-mode CTS experiments at ASDEX Upgrade [6]. 


\subsection{Electrostatic Daughter Waves at the UHR}

To study PDIs near the UHR we derive dispersion relations for the electrostatic daughter waves likely to be involved in such instabilities. First, we note that, when $\left|\left(\operatorname{Re}(\omega)-n \omega_{c e}\right) /\left(k_{z} v_{T e}\right)\right|>2$ (i.e. $\operatorname{Re}(\omega) \not \approx n \omega_{c e}$, for all $\left.n \in \mathbb{Z}\right), b_{e}=k_{\perp}^{2} r_{L e}^{2} \ll 1$, $k_{z}^{2} / k_{\perp}^{2} \ll 1$, and $\nu_{e} / \operatorname{Re}(\omega) \ll 1$, the electron $\chi_{\sigma}(\mathbf{k}, \omega)$ in $(9)$ may be approximated by [1]

$$
\begin{aligned}
\chi_{e}(\mathbf{k}, \omega) \approx & -\frac{\omega_{p e}^{2}}{\omega^{2}-\omega_{c e}^{2}}-\frac{3 \omega_{p e}^{2} \omega_{c e}^{2} b_{e}}{\left(\omega^{2}-\omega_{c e}^{2}\right)\left(\omega^{2}-4 \omega_{c e}^{2}\right)} \\
& +\frac{\omega_{p e}^{2}}{\omega^{2}}\left[\frac{\omega_{c e}^{2}}{\omega^{2}-\omega_{c e}^{2}}+\mathrm{i} 2 \sqrt{\pi} \frac{\omega^{3}}{\left|k_{z}\right|^{3} v_{T e}^{3}} \mathrm{e}^{\left.-\omega^{2} /\left(k_{z}^{2} v_{T e}^{2}\right)\right]} \frac{k_{z}^{2}}{k_{\perp}^{2}}+\mathrm{i} \frac{\omega_{p e}^{2}\left(\omega^{2}+\omega_{c e}^{2}\right)}{\left(\omega^{2}-\omega_{c e}^{2}\right)^{2}} \frac{\nu_{e}}{\omega} .\right.
\end{aligned}
$$

For the high-frequency daughter waves, the ion response is negligible and the dispersion relation, $\operatorname{Re}\left[\epsilon\left(-\mathbf{k}, \omega_{2}\right)\right] \approx 1+\operatorname{Re}\left[\chi_{e}\left(-\mathbf{k}, \omega_{2}\right)\right]=0$, may be written as

$$
1-\frac{\omega_{p e}^{2}}{\omega_{2}^{2}-\omega_{c e}^{2}}-\frac{3 \omega_{p e}^{2} \omega_{c e}^{2} b_{e}}{\left(\omega_{2}^{2}-\omega_{c e}^{2}\right)\left(\omega_{2}^{2}-4 \omega_{c e}^{2}\right)}+\frac{\omega_{p e}^{2} \omega_{c e}^{2}}{\omega_{2}^{2}\left(\omega_{2}^{2}-\omega_{c e}^{2}\right)} \frac{k_{z}^{2}}{k_{\perp}^{2}}=0 .
$$

This is a cubic equation for $\omega_{2}^{2}$ in terms of $\mathbf{k}$ and the plasma parameters which can be solved using standard algebraic techniques. However, for the cases of interest the terms involving $b_{e}$ and $k_{z}^{2} / k_{\perp}^{2}$ are small. Ignoring these, the order 0 dispersion relation becomes $\omega_{2}^{2} \approx \omega_{U H}^{2}$, i.e. the dispersion relation gives a non-propagating oscillation at the UH frequency. Expanding (34) to order 1 in $b_{e}$ and $k_{z}^{2} / k_{\perp}^{2}$ around $\omega_{2}=\omega_{U H}$, we find

$$
\omega_{2}^{2} \approx \omega_{U H}^{2}-\omega_{p e}^{2}\left[\frac{b_{e}}{1-\omega_{p e}^{2} /\left(3 \omega_{c e}^{2}\right)}+\frac{\omega_{c e}^{2}}{\omega_{U H}^{2}} \frac{k_{z}^{2}}{k_{\perp}^{2}}\right] .
$$

The above dispersion relation describes the previously mentioned limiting form of electron Bernstein waves known as warm UH waves, since $\omega_{2}$ is close to $\omega_{U H}$ and depends on $T_{e}$ through $b_{e}$, which are the principal high-frequency daughter waves in PDIs near the UHR. It is similar to the dispersion relation used by [1,21,22] to study PDIs near the UHR, and reduces to the one used by [4] for $k_{z}=0$ and the one used by [2] for $\omega_{p e}^{2} \ll \omega_{c e}^{2}$. The dispersion relation has a cut-off $(k=0)$ at $\omega_{2}=\omega_{U H}$, and propagating waves only exist for $\omega_{2}<\omega_{U H}$ when $\omega_{p e}^{2}<3 \omega_{c e}^{2}$, while they exist for $\omega_{2}>\omega_{U H}$ when $\omega_{p e}^{2}>3 \omega_{c e}^{2}$ and $k_{z}=0$. Thus, we see that a PDI in which the X-mode pump wave (with $\omega_{0}<\omega_{U H}$ ) decays directly to two electrostatic daughter waves, as we are considering, will only be feasible when $\omega_{p e}^{2}<3 \omega_{c e}^{2}$, limiting our theory to underdense and weakly overdense plasmas $\left(\omega_{p e} /\left|\omega_{c e}\right|<\sqrt{3}\right)$, which are found in conventional tokamaks, as well as many stellarator experiments. When $\omega_{p e}^{2}>3 \omega_{c e}^{2}$, as in spherical tokamaks or most ionospheric studies, it is necessary to consider PDIs for the linearly converted warm UH waves themselves, which requires consideration of the backscattering type of instability [17. The above dispersion relation is invalid when $\omega_{p e}^{2} \approx 3 \omega_{c e}^{2}$, where the UHR coincides with the 2nd harmonic ECR, $\omega_{2} \approx \omega_{U H} \approx 2\left|\omega_{c e}\right|$, violating the assumptions used for deriving (35); in fact, PDIs may be inhibited in a narrow band around $\omega_{U H}=n\left|\omega_{c e}\right|$ for all $n \in \mathbb{N}$ due to bandgap effects, as observed in ionospheric modification experiments [23]. 
For reference we note that the group velocity of the warm UH waves, given by $\mathbf{v}_{g}\left(\mathbf{k}, \omega_{2}\right)=\left[\partial \omega_{2}^{2}(\mathbf{k}) / \partial \mathbf{k}\right] /\left(2 \omega_{2}\right)$, may be found by exploiting the azimuthal symmetry of (35) in $\mathbf{k}$-space,

$$
\mathbf{v}_{g}\left(-\mathbf{k}, \omega_{2}\right)=\frac{\omega_{p e}^{2}}{k_{\perp} \omega_{2}}\left\{\left[\frac{b_{e}}{1-\omega_{p e}^{2} /\left(3 \omega_{c e}^{2}\right)}-\frac{\omega_{c e}^{2}}{\omega_{U H}^{2}} \frac{k_{z}^{2}}{k_{\perp}^{2}}\right] \mathbf{e}_{\perp}+\frac{\omega_{c e}^{2}}{\omega_{U H}^{2}} \frac{k_{z}}{k_{\perp}} \mathbf{e}_{z}\right\}
$$

where $\mathbf{e}_{\perp}=\mathbf{k}_{\perp} / k_{\perp}$. The relatively small value of $v_{g}\left(-\mathbf{k}, \omega_{2}\right)$, especially for $k_{z}=0$, is important for reducing the PDI threshold in inhomogeneous plasmas. Note also that, even though $k_{z}^{2} / k_{\perp}^{2} \ll 1, \mathbf{v}_{g}\left(-\mathbf{k}, \omega_{2}\right)$ may have a significant $z$-component for $k_{z} \neq 0$. Finally, we calculate $\Gamma\left(-\mathbf{k}, \omega_{2}\right) \approx \operatorname{Im}\left[\chi_{e}\left(-\mathbf{k}, \omega_{2}\right)\right] /\left.\left\{\partial \operatorname{Re}\left[\chi_{e}(-\mathbf{k}, \omega)\right] / \partial \omega\right\}\right|_{\omega=\omega_{2}}$ using the lowest order approximation, $\partial \operatorname{Re}\left[\chi_{e}(\mathbf{k}, \omega)\right] / \partial \omega \approx 2 \omega \omega_{p e}^{2} /\left(\omega^{2}-\omega_{c e}^{2}\right)^{2}$, from (33),

$$
\Gamma\left(-\mathbf{k}, \omega_{2}\right) \approx \sqrt{\pi} \frac{\left(\omega_{2}^{2}-\omega_{c e}^{2}\right)^{2}}{\left|k_{z}\right| k_{\perp}^{2} v_{T e}^{3}} \mathrm{e}^{-\omega_{2}^{2} /\left(k_{z}^{2} v_{T e}^{2}\right)}+\frac{\omega_{2}^{2}+\omega_{c e}^{2}}{2 \omega_{2}^{2}} \nu_{e} \approx \frac{\omega_{2}^{2}+\omega_{c e}^{2}}{2 \omega_{2}^{2}} \nu_{e}
$$

the last approximation follows from the fact that $\omega_{2}^{2} /\left(k_{z}^{2} v_{T e}^{2}\right)$ is generally a large quantity, making the first (Landau damping) term extremely small and showing that warm UH waves are mainly damped by collisions; Landau damping is included due to its possible importance in damping the low-frequency daughter waves discussed below.

There is a larger number of possible low-frequency electrostatic daughter waves which may be excited by PDIs near the UHR, since the bulk of the frequency shift necessary to satisfy the selection rule is provided by the warm UH wave. However, because the low-frequency daughter waves should also be able to satisfy the k-selection rules, we confine our attention to the cases where (33) is still a valid approximation for $\chi_{e}(\mathbf{k}, \omega)$, taking the limit $|\omega|^{2} \ll \omega_{c e}^{2}$,

$\chi_{e}(\mathbf{k}, \omega) \approx \frac{\omega_{p e}^{2}}{\omega_{c e}^{2}}-\frac{3 \omega_{p e}^{2}}{4 \omega_{c e}^{2}} b_{e}+\frac{\omega_{p e}^{2}}{\omega^{2}}\left[-1+\mathrm{i} 2 \sqrt{\pi} \frac{\omega^{3}}{\left|k_{z}\right|^{3} v_{T e}^{3}} \mathrm{e}^{-\omega^{2} /\left(k_{z}^{2} v_{T e}^{2}\right)}\right] \frac{k_{z}^{2}}{k_{\perp}^{2}}+\mathrm{i} \frac{\omega_{p e}^{2}}{\omega_{c e}^{2}} \frac{\nu_{e}}{\omega}$.

Note that this expression imposes a very strict condition on the smallness of $k_{z}^{2} / k_{\perp}^{2}$ (since generally $\omega_{p e}^{2} /|\omega|^{2} \gg 1$ ) and that it does not satisfy the low-frequency quasi-neutrality condition, $\left|\chi_{e}\left(\mathbf{k}, \omega_{1}\right)\right| \gg 1$, which is assumed by [35]. In general, $\chi_{i}(\mathbf{k}, \omega)$ should be determined from an expression like $(9]$, but $\nu_{i} / \nu_{e}=\mathrm{O}\left(\sqrt{m_{e} / m_{i}}\right) \ll 1[51]$, so we can ignore collisions completely for the ions. It is, however, not possible to expand $\chi_{i}(\mathbf{k}, \omega)$ in $b_{i}=r_{L i}^{2} k_{\perp}^{2}$, as was done for $\chi_{e}(\mathbf{k}, \omega)$ in $b_{e}$, since $r_{L i}^{2} / r_{L e}^{2}=m_{i} T_{i} /\left(Z_{i}^{2} m_{e} T_{e}\right) \gg 1$, which implies that $b_{i} \gg b_{e} \ll 1$, not imposing any particular restriction on $b_{i}$. One can assume $b_{i}$ to be small, as was done by [4], but generally it is necessary to evaluate (9), with $\nu_{i} \approx 0$ and $k_{z}^{2} / k_{\perp}^{2} \ll 1$, including a large number of terms from the sum over $n$ due to the potentially large value of $b_{i}$, in order to obtain $\chi_{i}(\mathbf{k}, \omega)$. If this is combined with $\chi_{e}(\mathbf{k}, \omega)$ from (38), the dispersion relation for pure ion Bernstein waves, which has a root between each integer value of $\omega_{c i}$, is obtained. The present paper is mainly concerned with highorder pure ion Bernstein waves, leading to frequency shifts on the order of $\omega_{p i}(\sim 1 \mathrm{GHz}$ in the ASDEX Upgrade main plasma), where $\chi_{i}(\mathbf{k}, \omega)$ may be evaluated in the limit of $\operatorname{Re}(\omega) \gg \omega_{c i}$ and $b_{i} \gg 1$; remember that $\omega_{p i}^{2} / \omega_{c i}^{2}=\left[m_{i} /\left(Z_{i} m_{e}\right)\right] \omega_{p e}^{2} / \omega_{c e}^{2}$ in a simple plasma, so $\omega_{c i} \ll \omega_{p i}$ for $\omega_{p e} \sim\left|\omega_{c e}\right|$. Here, the basic behaviour can be obtained by 
considering the ions to be unmagnetised, since their trajectories are essentially straight lines on the relevant temporal and spatial scales (respectively, $1 / \omega_{1} \sim 1 / \omega_{p i} \sim 200 \mathrm{ps}$ and $1 / k_{\perp} \sim r_{L e} / \sqrt{b_{e}} \sim 30 \mu \mathrm{m}$ near the UHR in the considered ASDEX Upgrade discharge), although the details at integer values of $\omega_{c i}$ and interpretation of the Landau damping involve some subtleties [51,52. For unmagnetised ions we may insert $\chi_{\sigma}(\mathbf{k}, \omega)$ from 10 with $\nu_{\sigma} \approx 0$, and find

$$
\begin{aligned}
\chi_{i}(\mathbf{k}, \omega) & \approx \frac{2 \omega_{p i}^{2}}{k^{2} v_{T i}^{2}}\left[1+\frac{\omega}{k v_{T i}} Z\left(\frac{\omega}{k v_{T i}}\right)\right] \approx-\frac{\omega_{p i}^{2}}{\omega^{2}}\left[1+\frac{3 k^{2} v_{T i}^{2}}{2 \omega^{2}}-\mathrm{i} 2 \sqrt{\pi} \frac{\omega^{3}}{k^{3} v_{T i}^{3}} \mathrm{e}^{-\omega^{2} /\left(k^{2} v_{T i}^{2}\right)}\right] \\
& \approx-\frac{\omega_{p i}^{2}}{\omega^{2}}\left[1+\frac{3 T_{i}}{Z_{i} T_{e}} \frac{\left|\omega_{c e}\right| \omega_{c i}}{\omega^{2}} b_{e}-\mathrm{i} 2 \sqrt{\pi} \frac{\omega^{3}}{k_{\perp}^{3} v_{T i}^{3}} \mathrm{e}^{-\omega^{2} /\left(k_{\perp}^{2} v_{T i}^{2}\right)}\right]
\end{aligned}
$$

where the first approximation follows from an asymptotic expansion of $Z$, valid for $\operatorname{Re}(\omega) /\left(k v_{T e}\right)>\sqrt{2}[1]$, and the second approximation follows from setting $k \approx k_{\perp}$, which is permissible due to the requirement $k_{z}^{2} / k_{\perp}^{2} \lesssim \omega_{p i}^{2} / \omega_{p e}^{2} \ll 1$, imposed by the use of $\chi_{e}(\mathbf{k}, \omega)$ from $(38)$ for $|\omega| \sim \omega_{p i}$. Note that $\left|\omega_{c e}\right| \omega_{c i} \sim \omega_{p i}^{2}$ for $\omega_{p e} \sim\left|\omega_{c e}\right|$, ensuring that the $b_{e}$-term in (39) is indeed of order $b_{e} \ll 1$, unless $\omega_{p e} \ll\left|\omega_{c e}\right|$ or $T_{i} \gg Z_{i} T_{e}$. We now obtain the dispersion relation of the low-frequency daughter waves in a simple plasma, $\operatorname{Re}\left[\epsilon\left(\mathbf{k}, \omega_{1}\right)\right]=1+\operatorname{Re}\left[\chi_{i}\left(\mathbf{k}, \omega_{1}\right)\right]+\operatorname{Re}\left[\chi_{e}\left(\mathbf{k}, \omega_{1}\right)\right]=0$, by inserting $\chi_{e}(\mathbf{k}, \omega)$ from (38) and $\chi_{i}(\mathbf{k}, \omega)$ from 39 ,

$$
1+\frac{\omega_{p e}^{2}}{\omega_{c e}^{2}}-\frac{\omega_{p i}^{2}}{\omega_{1}^{2}}-3\left(\frac{\omega_{p e}^{2}}{4 \omega_{c e}^{2}}+\frac{T_{i}}{Z_{i} T_{e}} \frac{\omega_{p i}^{2}\left|\omega_{c e}\right| \omega_{c i}}{\omega_{1}^{4}}\right) b_{e}-\frac{\omega_{p e}^{2}}{\omega_{1}^{2}} \frac{k_{z}^{2}}{k_{\perp}^{2}}=0 .
$$

This quadratic equation for $\omega_{1}^{2}$ in terms of $b_{e}$ and $k_{z}^{2} / k_{\perp}^{2}$ can be solved by the standard approach. However, just as for the high-frequency daughter waves, we note that the $b_{e}$ and $k_{z}^{2} / k_{\perp}^{2}$-terms represent corrections, and that, ignoring these, the order 0 solution is $\omega_{1}^{2} \approx \omega_{c e}^{2} \omega_{p i}^{2} / \omega_{U H}^{2}=\omega_{L H}^{2}$, i.e. a non-propagating oscillation at the LH frequency, $\omega_{L H}$, which is a resonance for cold electromagnetic plasma waves. Now, expanding 40 to order 1 in $b_{e}$ and $k_{z}^{2} / k_{\perp}^{2}$ around $\omega_{1}=\omega_{L H}$, also using the simple plasma identities $\omega_{p e}^{2} / \omega_{p i}^{2}=m_{i} /\left(Z_{i} m_{e}\right)$ and $\left|\omega_{c e}\right| \omega_{c i} / \omega_{L H}^{2}=\omega_{U H}^{2} / \omega_{p e}^{2}$, the dispersion relation of the lowfrequency daughter waves is found to be,

$$
\omega_{1}^{2} \approx \omega_{L H}^{2}\left[1+3\left(\frac{\omega_{p e}^{2}}{4 \omega_{U H}^{2}}+\frac{T_{i}}{Z_{i} T_{e}} \frac{\omega_{U H}^{2}}{\omega_{p e}^{2}}\right) b_{e}+\frac{m_{i}}{Z_{i} m_{e}} \frac{k_{z}^{2}}{k_{\perp}^{2}}\right],
$$

which describes so-called warm LH waves. The above dispersion relation was obtained in [1] and is similar to the one used by [2] for $\omega_{p e}^{2} \ll \omega_{c e}^{2}$ and the electrostatic version given by [52] for $\omega_{p e}^{2} \gg \omega_{c e}^{2}$. Evidently, the warm LH waves have a cut-off at $\omega_{1}=\omega_{L H}$ and propagating waves only exist for $\omega_{1}>\omega_{L H}$, meaning that the frequency shift of the high-frequency daughter waves for PDIs with warm LH low-frequency daughter waves should always exceed $\omega_{L H}$. It is also clear that the above dispersion relation requires $k_{z}^{2} / k_{\perp}^{2} \lesssim Z_{i} m_{e} / m_{i} \ll 1$, which is a strict requirement on the smallness of $k_{z}^{2} / k_{\perp}^{2}$ indeed. Following [1 3], we shall employ (41) when discussing both the resonant and the nonresonant PDIs. However, in the non-resonant case the mode leading to the largest $\gamma$, or the largest $G$, may deviate somewhat from the one given by the above dispersion relation, as the underlying assumption of $\left|\operatorname{Im}\left[\epsilon\left(\mathbf{k}, \omega_{1}\right)\right]\right| \ll\left|\operatorname{Re}\left[\epsilon\left(\mathbf{k}, \omega_{1}\right)\right]\right|$ is not generally 
satisfied here. This effect should not significantly change the results of the following analysis, and we further note that finding an unstable mode, even if it is not the most unstable one, will still imply the existence of a PDI, thus giving an upper bound of the PDI threshold. Nevertheless, a detailed study of the dispersion relation in the nonresonant case, and its consequences for the predicted $\omega_{1}$, may be of some interest for future work.

As was done for the warm UH waves, we compute the group velocity, $\mathbf{v}_{g}\left(\mathbf{k}, \omega_{1}\right)=$ $\left[\partial \omega_{1}^{2}(\mathbf{k}) / \partial \mathbf{k}\right] /\left(2 \omega_{1}\right)$, of the warm LH waves,

$\mathbf{v}_{g}\left(\mathbf{k}, \omega_{1}\right)=\frac{\omega_{L H}^{2}}{k_{\perp} \omega_{1}}\left\{\left[3\left(\frac{\omega_{p e}^{2}}{4 \omega_{U H}^{2}}+\frac{T_{i}}{Z_{i} T_{e}} \frac{\omega_{U H}^{2}}{\omega_{p e}^{2}}\right) b_{e}-\frac{m_{i}}{Z_{i} m_{e}} \frac{k_{z}^{2}}{k_{\perp}^{2}}\right] \mathbf{e}_{\perp}+\frac{m_{i}}{Z_{i} m_{e}} \frac{k_{z}}{k_{\perp}} \mathbf{e}_{z}\right\}$.

Similar to $\mathbf{v}_{g}\left(-\mathbf{k}, \omega_{2}\right), \mathbf{v}_{g}\left(\mathbf{k}, \omega_{1}\right)$ is relatively small for $k_{z}=0$, resulting in a low resonant PDI threshold in inhomogeneous plasmas, but acquires a significant $z$-component for $k_{z} \neq 0$, even when $k_{z}^{2} / k_{\perp}^{2} \ll Z_{i} m_{e} / m_{i}$. Note also that $\mathbf{v}_{g}\left(\mathbf{k}, \omega_{1}\right)$ and $\mathbf{v}_{g}\left(-\mathbf{k}, \omega_{2}\right)$ are parallel for $k_{z}=0$ and $\omega_{p e}^{2}<3 \omega_{c e}^{2}$, making the backscattering instability impossible in this case. We finally calculate $\Gamma\left(\mathbf{k}, \omega_{1}\right)=\operatorname{Im}\left[\epsilon\left(\mathbf{k}, \omega_{1}\right)\right] /\left.\{\partial \operatorname{Re}[\epsilon(\mathbf{k}, \omega)] / \partial \omega\}\right|_{\omega=\omega_{1}}$, using that $\partial \operatorname{Re}[\epsilon(\mathbf{k}, \omega)] / \partial \omega \approx \partial \operatorname{Re}\left[\chi_{i}(\mathbf{k}, \omega)\right] / \partial \omega \approx 2 \omega_{p i}^{2} / \omega^{3}$,

$\Gamma\left(\mathbf{k}, \omega_{1}\right) \approx \sqrt{\pi} \frac{\omega_{1}^{2}}{k_{\perp}^{2} v_{T i}^{2}}\left[\frac{\omega_{1}}{k_{\perp} v_{T i}} \mathrm{e}^{-\omega_{1}^{2} /\left(k_{\perp}^{2} v_{T i}^{2}\right)}+\frac{T_{i}}{Z_{i} T_{e}} \frac{\omega_{1}}{\left|k_{z}\right| v_{T e}} \mathrm{e}^{-\omega_{1}^{2} /\left(k_{z}^{2} v_{T e}^{2}\right)}\right] \omega_{1}+\frac{\omega_{p e}^{2}}{2 \omega_{c e}^{2}} \frac{\omega_{1}^{2}}{\omega_{p i}^{2}} \nu_{e},($

which shows that warm LH waves are subject to ion Landau damping (first term), electron Landau damping (second term), and electron collisional damping (third term).

\subsection{Parametric Decay into Warm UH and Warm LH Waves}

We now have all the ingredients necessary for studying PDIs in which the X-mode pump wave decays into a warm UH wave and a warm LH wave near the UHR. We restrict our attention to cases with $k_{z}=0$ since a small, but finite, $k_{z}^{2} / k_{\perp}^{2}$ will rapidly increase convective losses parallel to $\mathbf{B}$ without altering the propagation perpendicular to $\mathbf{B}$ appreciably; a discussion including finite $k_{z}$ within the dipole approximation is found in [1]; if the dipole approximation is abandoned, at least one of the daughter waves must have finite $\left|k_{z}\right| \sim\left|k_{0 z}\right| \lesssim 3 \mathrm{~cm}^{-1}\left(k_{z}^{2} / k_{\perp}^{2} \lesssim 10^{-4}\right)$ to satisfy the selection rules in the ASDEX Upgrade discharge under consideration, but this should not significantly alter the results of the following analysis in the nonresonant case [2] or in the resonant case, if $k_{z} \approx k_{0 z}$ for the warm UH wave here. Our goal is first to evaluate $b_{e}$ from the selection rules, to determine the conditions under which the PDI is resonant and non-resonant, and the limits of validity for this analytical approach. In order to allow easier manipulation, we rewrite (41) and (35) as $\omega_{1}=\omega_{L H} \sqrt{1+A_{1} b_{e}} \approx \omega_{L H}\left(1+A_{1} b_{e} / 2\right)$ and $\omega_{2}^{2}=\omega_{U H}^{2}-\omega_{p e}^{2} A_{2} b_{e}$, with $A_{1}=3\left\{\omega_{p e}^{2} /\left(4 \omega_{U H}^{2}\right)+\left[T_{i} /\left(Z_{i} T_{e}\right)\right] \omega_{U H}^{2} / \omega_{p e}^{2}\right\}$ and $A_{2}=1 /\left[1-\omega_{p e}^{2} /\left(3 \omega_{c e}^{2}\right)\right]$, respectively. Then, from the selection rules, $\omega_{2}^{2}=\left(\omega_{0}-\omega_{1}\right)^{2} \approx\left(\omega_{0}-\omega_{L H}\right)^{2}-A_{1}\left(\omega_{0}-\omega_{L H}\right) \omega_{L H} b_{e}$, and we find

$$
b_{e} \approx \frac{\omega_{U H}^{2}-\left(\omega_{0}-\omega_{L H}\right)^{2}}{A_{2} \omega_{p e}^{2}-A_{1}\left(\omega_{0}-\omega_{L H}\right) \omega_{L H}} \approx \frac{\omega_{U H}^{2}-\omega_{0}^{2}+2 \omega_{0} \omega_{L H}}{A_{2} \omega_{p e}^{2}-A_{1} \omega_{0} \omega_{L H}}
$$


where the last approximation follows by neglecting the $\omega_{L H}^{2}$-terms, since $\omega_{L H} \ll \omega_{0}, \omega_{p e}$. Clearly, the assumption that $b_{e} \ll 1$ is only valid for $\omega_{0}^{2} \approx \omega_{U H}^{2}$, i.e. near the UHR of the gyrotron, where 44 gives $b_{e}=\mathrm{O}\left(\omega_{0} \omega_{L H} / \omega_{p e}^{2}\right) \ll 1$; however, even here problems arise for $\omega_{p e}^{2} \ll \omega_{c e}^{2}$, as $\omega_{0} \gg \omega_{p e}$ and $A_{1} \gg T_{i} /\left(Z_{i} T_{e}\right)$ in this case. To quantify the limits of validity of the above theory, as well as the conditions under which the resonant and non-resonant PDIs occur, we calculate,

$\zeta_{i}^{2}=\frac{\omega_{1}^{2}}{k_{\perp}^{2} v_{T i}^{2}}=\frac{Z_{i} T_{e}}{2 T_{i}} \frac{\omega_{1}^{2}}{\omega_{c i}\left|\omega_{c e}\right| b_{e}} \approx \frac{Z_{i} T_{e}}{2 T_{i}} \frac{\omega_{p e}^{2}}{\omega_{U H}^{2}}\left(\frac{A_{2} \omega_{p e}^{2}-A_{1} \omega_{0} \omega_{L H}}{\omega_{U H}^{2}-\omega_{0}^{2}+2 \omega_{0} \omega_{L H}}+A_{1}\right)$,

since this parameter determines the relative importance of the real and imaginary parts of the ion susceptibility, $\chi_{i}\left(\mathbf{k}, \omega_{1}\right) \approx\left(\omega_{p i}^{2} / \omega_{1}^{2}\right)\left[-1-3 /\left(2 \zeta_{i}^{2}\right)+\mathrm{i} L\left(\zeta_{i}\right)\right]$ with $L\left(\zeta_{i}\right)=2 \sqrt{\pi} \zeta_{i}^{3} \mathrm{e}^{-\zeta_{i}^{2}}$, and the limit of the validity of this approximate form through the requirement $\zeta_{i}^{2}>2$; the non-resonant $\mathrm{PDI}$ occurs for $L\left(\zeta_{i}\right) \sim 1$, while the resonant PDI occurs for $L\left(\zeta_{i}\right) \ll 1$. A simple expression for $\zeta_{i}^{2}$ in terms of the plasma parameters is obtained by setting $\omega_{0} \approx \omega_{U H}$ in 45 ,

$\zeta_{i}^{2} \approx \frac{Z_{i} T_{e}}{4 T_{i}} \frac{\omega_{p e}^{2}}{\omega_{U H}^{2}}\left(\frac{A_{2} \omega_{p e}^{2}}{\omega_{U H} \omega_{L H}}+A_{1}\right) \approx \frac{Z_{i} T_{e}}{4 T_{i}} \sqrt{\frac{m_{i}}{Z_{i} m_{e}}} \frac{\omega_{p e}^{3} /\left|\omega_{c e}\right|^{3}}{\left(1+\omega_{p e}^{2} / \omega_{c e}^{2}\right)\left[1-\omega_{p e}^{2} /\left(3 \omega_{c e}^{2}\right)\right]}+\frac{3}{4}$

the last approximation follows from the identity $\omega_{p e}^{2} /\left(\omega_{U H} \omega_{L H}\right)=\sqrt{m_{i} /\left(Z_{i} m_{e}\right)} \omega_{p e} /\left|\omega_{c e}\right|$ and neglect of the first term of $A_{1}=3\left\{\omega_{p e}^{2} /\left(4 \omega_{U H}^{2}\right)+\left[T_{i} /\left(Z_{i} T_{e}\right)\right] \omega_{U H}^{2} / \omega_{p e}^{2}\right\}$ in comparison with $A_{2} \omega_{p e}^{2} /\left(\omega_{U H} \omega_{L H}\right)$, while the second term of $A_{1}$ is kept due to its importance at small $\omega_{p e} /\left|\omega_{c e}\right|$. A contour plot of $L\left(\zeta_{i}\right)$ and the line at which $\zeta_{i}^{2}=2$ for a deuterium-like plasma, $m_{i} /\left(Z_{i} m_{e}\right)=3.67 \times 10^{3}$, versus $\omega_{p e} /\left|\omega_{c e}\right|$ and $Z_{i} T_{e} / T_{i}$ is seen in figure 7 . From the figure, it is clear that the PDI will be non-resonant for moderate values of $\omega_{p e} /\left|\omega_{c e}\right|$ and $Z_{i} T_{e} / T_{i}$, resonant for large values, and that the analytical approximation breaks down for sufficiently small values. For $t \geq 2.900 \mathrm{~s}$ in ASDEX Upgrade discharge 28286, the UHR is encountered at $\omega_{p e} /\left|\omega_{c e}\right| \approx 0.5$ by the CTS probe rays, and the plasma is further modelled as a pure deuterium plasma $\left(Z_{i}=1, m_{i} / m_{e}=3.67 \times 10^{3}\right)$ with $T_{i}=T_{e}$, placing the expected PDI in the non-resonant region of figure 7 .

Now that the basic questions related to the selection rules have been answered, we turn our attention to the problem of determining $\gamma$ and the PDI threshold conditions for the non-resonant and resonant cases. Except when determining $b_{e}$ and $\zeta_{i}$, the lowest order approximations $\left(\omega_{1} \approx \omega_{L H}\right.$ and $\left.\omega_{2} \approx \omega_{U H}\right)$ are used and the $b_{e}$-terms neglected, resulting in the following approximate expressions,

$$
\begin{gathered}
\chi_{i}\left(\mathbf{k}, \omega_{1}\right) \approx \frac{\omega_{U H}^{2}}{\omega_{c e}^{2}}\left[-1+\mathrm{i} L\left(\zeta_{i}\right)\right], 1+\chi_{e}\left(\mathbf{k}, \omega_{1}\right) \approx \frac{\omega_{U H}^{2}}{\omega_{c e}^{2}}\left(1+\mathrm{i} C_{e}\right), \\
\epsilon\left(\mathbf{k}, \omega_{1}\right) \approx \mathrm{i} \frac{\omega_{U H}^{2}}{\omega_{c e}^{2}}\left[L\left(\zeta_{i}\right)+C_{e}\right],\left.\quad \frac{\partial \operatorname{Re}[\epsilon(\mathbf{k}, \omega)]}{\partial \omega}\right|_{\omega=\omega_{1}} \approx \frac{2 \omega_{U H}^{2}}{\omega_{c e}^{2} \omega_{L H}},\left.\quad \frac{\partial \operatorname{Re}[\epsilon(-\mathbf{k}, \omega)]}{\partial \omega}\right|_{\omega=\omega_{2}} \frac{2 \omega_{U H}}{\omega_{p e}^{2}},
\end{gathered}
$$

where $C_{e}=\left(\omega_{p e}^{2} / \omega_{U H}^{2}\right) \nu_{e} / \omega_{L H} \ll 1$. Note that $\left|\epsilon\left(\mathbf{k}, \omega_{1}\right)\right|$ is indeed small in the resonant case, $L\left(\zeta_{i}\right) \ll 1$, and non-negligible in the non-resonant case, $L\left(\zeta_{i}\right) \sim 1$. An approximate version of $\mu_{e}^{2}$ may also be obtained from (14) by noting that the X-mode is a quasielectrostatic cold UH wave, and thus linearly polarised, near the UHR $\left(\omega_{0} \approx \omega_{U H}\right)$. 


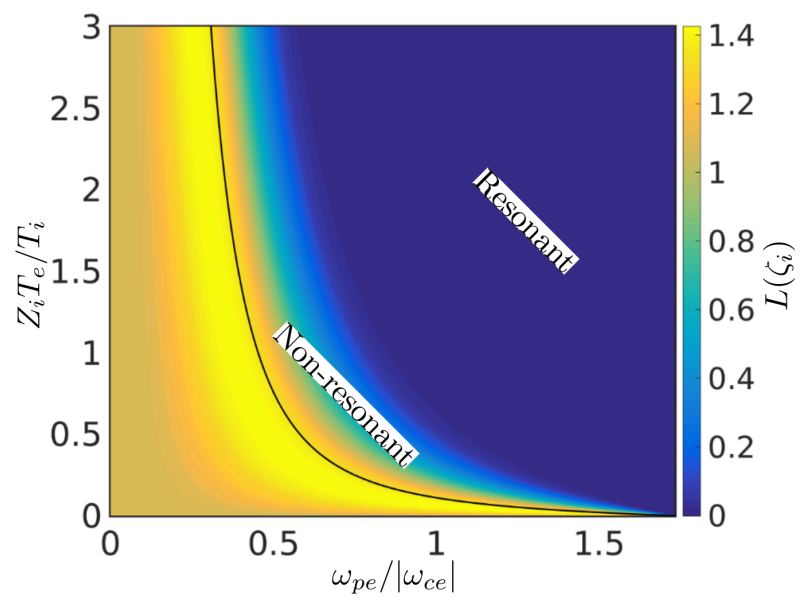

Figure 7. Contour plot of $L\left(\zeta_{i}\right)$ and the black line at which $\zeta_{i}^{2}=2$ versus $\omega_{p e} /\left|\omega_{c e}\right|$ and $Z_{i} T_{e} / T_{i}$, with $\zeta_{i}^{2}$ from 46 for a deuterium-like plasma, $m_{i} /\left(Z_{i} m_{e}\right)=3.67 \times 10^{3}$. Areas where the PDI is resonant and non-resonant are marked with text boxes. The analytical approximation breaks down below the $\zeta_{i}^{2}=2$ line. In the experiment $\omega_{p e} /\left|\omega_{c e}\right| \approx 0.5$ and $Z_{i} T_{e} / T_{i} \approx 1$, placing the PDI in the non-resonant region.

From this it follows that all components of $\mathbf{E}_{0}$ have the same phase, and further taking $\mathbf{k}=k_{\perp} \mathbf{e}_{x}$, we find

$\mu_{e}^{2} \approx \frac{e^{2} k_{\perp}^{2}}{m_{e}^{2}\left(\omega_{0}^{2}-\omega_{c e}^{2}\right)^{2}}\left(\left|E_{0 x}\right|^{2}+\frac{\omega_{c e}^{2}}{\omega_{0}^{2}}\left|E_{0 y}\right|^{2}\right) \approx b_{e} \frac{\omega_{c e}^{2}}{\omega_{p e}^{2}}\left(\frac{\epsilon_{0}\left|E_{0 x}\right|^{2}}{N_{e}^{(0)} T_{e}}+\frac{\omega_{c e}^{2}}{\omega_{U H}^{2}} \frac{\epsilon_{0}\left|E_{0 y}\right|^{2}}{N_{e}^{(0)} T_{e}}\right)$,

which is similar to the $\mu_{e}^{2}$ given by [2]. (48) shows that $\mu_{e}^{2}$ is generally a small quantity of order $b_{e} \ll 1$, as assumed when deriving the parametric dispersion relation; exceptions occur when $\omega_{p e}^{2} \ll \omega_{c e}^{2}$ or when the pump wave energy density $\left(\sim \epsilon_{0}\left|E_{0}\right|^{2}\right)$ is much larger than the thermal energy density $\left(\sim N_{e}^{(0)} T_{e}\right)$, and the weak coupling approximation breaks down, at the UHR. The above expression is clearly maximised for $\left|E_{0 x}\right|=\left|E_{0 \perp}\right|$, i.e. $\mathbf{E}_{0 \perp} \propto \mathbf{k}$, giving us the following $\mu_{e}^{2}$, useful for deriving the maximum $\gamma$,

$$
\mu_{e}^{2} \approx b_{e} \frac{\omega_{c e}^{2}}{\omega_{p e}^{2}} \frac{\epsilon_{0}\left|E_{0 \perp}\right|^{2}}{N_{e}^{(0)} T_{e}} .
$$

With (37), 47) and (49), we are now in a position to determine $\gamma$ for the non-resonant PDI from 28,

$\gamma \approx \frac{b_{e}}{8} \frac{1+L\left(\zeta_{i}\right) C_{e}}{L\left(\zeta_{i}\right)+C_{e}} \frac{\epsilon_{0}\left|E_{0 \perp}\right|^{2}}{N_{e}^{(0)} T_{e}} \omega_{U H}-\frac{\omega_{U H}^{2}+\omega_{c e}^{2}}{2 \omega_{U H}^{2}} \nu_{e} \approx \frac{b_{e}}{8 L\left(\zeta_{i}\right)} \frac{\epsilon_{0}\left|E_{0 \perp}\right|^{2}}{N_{e}^{(0)} T_{e}} \omega_{U H}-\frac{\omega_{U H}^{2}+\omega_{c e}^{2}}{2 \omega_{U H}^{2}} \nu_{e}$,

where $C_{e} \ll 1 \sim L\left(\zeta_{i}\right)$ has been used in the last approximation. From this we may easily determine the non-resonant PDI threshold in a homogeneous plasma $(\gamma>0)$,

$$
\left|E_{0 \perp}\right|^{2}>\frac{4 L\left(\zeta_{i}\right)}{b_{e}} \frac{\left(\omega_{U H}^{2}+\omega_{c e}^{2}\right) \nu_{e}}{\omega_{U H}^{3}} \frac{N_{e}^{(0)} T_{e}}{\epsilon_{0}},
$$

which is the generalisation of the expression given by [3] to finite $\omega_{p e} /\left|\omega_{c e}\right|$ and $L\left(\zeta_{i}\right) \neq 1$ obtained in [1] (the non-resonant $\gamma$, which is the one of relevance, is approximately $3 \mathrm{~ns}^{-1}$ near the UHR in the considered ASDEX Upgrade discharge). 
Noting that $\Gamma\left(\mathbf{k}, \omega_{1}\right) \approx\left[L\left(\zeta_{i}\right) \omega_{L H}+\left(\omega_{p e}^{2} / \omega_{U H}^{2}\right) \nu_{e}\right] / 2$, we can also determine $\gamma$ for the resonant PDI from (26),

$$
\gamma \approx \frac{1}{2}\left[\sqrt{\left(\frac{L\left(\zeta_{i}\right)}{2} \omega_{L H}-\frac{\omega_{c e}^{2}}{\omega_{U H}^{2}} \nu_{e}\right)^{2}+\frac{b_{e}}{4} \frac{\epsilon_{0}\left|E_{0 \perp}\right|^{2}}{N_{e}^{(0)} T_{e}} \omega_{U H} \omega_{L H}}-\frac{L\left(\zeta_{i}\right)}{2} \omega_{L H}-\nu_{e}\right],
$$

and the corresponding resonant PDI threshold in a homogeneous plasma,

$$
\left|E_{0 \perp}\right|^{2}>\frac{4\left[L\left(\zeta_{i}\right)+C_{e}\right]}{b_{e}} \frac{\left(\omega_{U H}^{2}+\omega_{c e}^{2}\right) \nu_{e}}{\omega_{U H}^{3}} \frac{N_{e}^{(0)} T_{e}}{\epsilon_{0}},
$$

which is very similar to that of the non-resonant PDI, but since we are now in the region of $L\left(\zeta_{i}\right) \ll 1$, its value is generally much smaller. For negligible damping, (52) becomes

$$
\gamma \approx \frac{1}{4} \sqrt{b_{e} \frac{\epsilon_{0}\left|E_{0 \perp}\right|^{2}}{N_{e}^{(0)} T_{e}} \omega_{U H} \omega_{L H}}
$$

which is similar to the $\gamma$ obtained by [4] for $\omega_{c i} \ll \omega_{p i}$, while being $\omega_{U H}^{2} / \omega_{p e}^{2}$ times that reported by [5, 27] within the weak coupling and dipole approximations, indicating that their approach reproduces a parametric dispersion relation similar to that of [18] here.

While the homogeneous PDI thresholds are of some interest, the actual thresholds are generally determined by inhomogeneities. For the resonant PDI well above the homogeneous threshold we can ignore damping and use $\gamma$ from (54), while the magnitudes of the group velocities of the daughter waves for $\mathbf{k} \perp \mathbf{B}$ become $v_{g}\left(\mathbf{k}, \omega_{1}\right) \approx$ $A_{1} b_{e} \omega_{L H} / k_{\perp}$ and $v_{g}\left(-\mathbf{k}, \omega_{2}\right) \approx A_{2} b_{e} \omega_{p e}^{2} /\left(\omega_{U H} k_{\perp}\right)$, such that 32 gives

$$
G \approx \frac{\pi}{8 A_{1} A_{2}} \frac{\omega_{U H}^{2}}{\omega_{p e}^{2}} \frac{l^{2}}{r_{L e}^{2}} \frac{\epsilon_{0}\left|E_{0 \perp}\right|^{2}}{N_{e}^{(0)} T_{e}}, \quad\left|E_{0 \perp}\right|^{2}>16 A_{1} A_{2} \frac{\omega_{p e}^{2}}{\omega_{U H}^{2}} \frac{r_{L e}^{2}}{l^{2}} \frac{N_{e}^{(0)} T_{e}}{\epsilon_{0}} ;
$$

the threshold is determined by the condition $G>2 \pi$, and all quantities are to be evaluated at $x=x_{r}$. Before an exact threshold can be obtained from the above expression, we need to determine

$\frac{1}{l^{2}}=\left|\left[\left.\left.\frac{\partial k_{x}\left(x, \omega_{1}\right)}{\partial x}\right|_{x=x_{r}} \frac{\partial k_{x}\left(x, \omega_{2}\right)}{\partial x}\right|_{x=x_{r}}\right]\right|=\frac{1}{2 \sqrt{b_{e} r_{L e}}}\left|\left[\left.\frac{\partial b_{e}\left(x, \omega_{1}\right)}{\partial x}\right|_{x=x_{r}}-\left.\frac{\partial b_{e}\left(x, \omega_{2}\right)}{\partial x}\right|_{x=x_{r}}\right]\right|$,

where the last equality follows from $k_{x}=k_{\perp}=\sqrt{b_{e}} / r_{L e}$ (for $\mathbf{k}=k_{\perp} \mathbf{e}_{x}$ ) and the fact that the selection rules are exactly satisfied at $x=x_{r}$. The $x$-derivatives of $b_{e}\left(x, \omega_{1}\right)=\left[\omega_{1}^{2}-\right.$ $\left.\omega_{L H}^{2}(x)\right] /\left[A_{1}(x) \omega_{L H}^{2}(x)\right]$ and $b_{e}\left(x, \omega_{2}\right)=\left[\omega_{U H}^{2}(x)-\omega_{2}^{2}\right] /\left[A_{2}(x) \omega_{p e}^{2}(x)\right]$ may be evaluated, neglecting terms proportional to the small quantities $\left[\omega_{1}^{2}-\omega_{L H}^{2}(x)\right] /\left[A_{1}(x) \omega_{L H}^{2}(x)\right]$ and $\left[\omega_{U H}^{2}(x)-\omega_{2}^{2}\right] /\left[A_{2}(x) \omega_{p e}^{2}(x)\right]$,

$\frac{\partial b_{e}\left(x, \omega_{1}\right)}{\partial x} \approx-\frac{1}{A_{1}(x) \omega_{L H}^{2}(x)} \frac{\mathrm{d} \omega_{L H}^{2}(x)}{\mathrm{d} x}=-\frac{1}{A_{1}(x)}\left[\frac{\omega_{c e}^{2}(x)}{\omega_{U H}^{2}(x)} \frac{1}{L_{N}(x)}+\frac{\omega_{p e}^{2}(x)}{\omega_{U H}^{2}(x)} \frac{2}{L_{B}(x)}\right]$,

$\frac{\partial b_{e}\left(x, \omega_{2}\right)}{\partial x} \approx \frac{1}{A_{2}(x) \omega_{p e}^{2}(x)} \frac{\mathrm{d} \omega_{U H}^{2}(x)}{\mathrm{d} x}=\frac{1}{A_{2}(x)}\left[\frac{1}{L_{N}(x)}+\frac{\omega_{c e}^{2}(x)}{\omega_{p e}^{2}(x)} \frac{2}{L_{B}(x)}\right]$ 
$L_{N}(x)=N_{e}^{(0)}(x) /\left[\mathrm{d} N_{e}^{(0)}(x) / \mathrm{d} x\right]=N_{i}^{(0)}(x) /\left[\mathrm{d} N_{i}^{(0)}(x) / \mathrm{d} x\right]$ and $L_{B}=B(x) /[\mathrm{d} B(x) / \mathrm{d} x]$ are the density and magnetic field strength gradient scale lengths, respectively. Plugging these expressions into (56), we find

$$
\frac{1}{l^{2}} \approx \frac{1}{\sqrt{b_{e}}}\left|\left(\frac{\omega_{c e}^{2}}{A_{1} \omega_{U H}^{2}}+\frac{1}{A_{2}}\right) \frac{1}{2 r_{L e} L_{N}}+\left(\frac{\omega_{p e}^{2}}{A_{1} \omega_{U H}^{2}}+\frac{\omega_{c e}^{2}}{A_{2} \omega_{p e}^{2}}\right) \frac{1}{r_{L e} L_{B}}\right|,
$$

and thus the resonant PDI threshold in an inhomogeneous plasma is

$$
\left|E_{0 \perp}\right|^{2}>\frac{8}{\sqrt{b_{e}}} \frac{\omega_{p e}^{2}}{\omega_{U H}^{2}}\left|\left(\frac{A_{2} \omega_{c e}^{2}}{\omega_{U H}^{2}}+A_{1}\right) \frac{r_{L e}}{L_{N}}+\left(\frac{A_{2} \omega_{p e}^{2}}{\omega_{U H}^{2}}+\frac{A_{1} \omega_{c e}^{2}}{\omega_{p e}^{2}}\right) \frac{2 r_{L e}}{L_{B}}\right| \frac{N_{e}^{(0)} T_{e}}{\epsilon_{0}} .
$$

For the non-resonant PDI well above the homogeneous threshold, we may neglect collisional damping and find $\gamma \approx\left\{b_{e} /\left[8 L\left(\zeta_{i}\right)\right]\right\}\left[\epsilon_{0}\left|E_{0 \perp}\right|^{2} /\left(N_{e}^{(0)} T_{e}\right)\right] \omega_{U H}$, while $v_{g}\left(-\mathbf{k}, \omega_{2}\right) \approx A_{2} b_{e} \omega_{p e}^{2} /\left(\omega_{U H} k_{\perp}\right)$ still holds, such that (31) yields

$$
G \approx \frac{k_{\perp} \ell}{4 A_{2} L\left(\zeta_{i}\right)} \frac{\omega_{U H}^{2}}{\omega_{p e}^{2}} \frac{\epsilon_{0}\left|E_{0 \perp}\right|^{2}}{N_{e}^{(0)} T_{e}}, \quad\left|E_{0 \perp}\right|^{2}>\frac{8 \pi A_{2} L\left(\zeta_{i}\right)}{k_{\perp} \ell} \frac{\omega_{p e}^{2}}{\omega_{U H}^{2}} \frac{N_{e}^{(0)} T_{e}}{\epsilon_{0}},
$$

where the threshold is again determined by the condition $G>2 \pi$. This is the generalisation of the non-resonant inhomogeneous PDI thresholds given by [2,3] obtained in [1]. By setting $\ell \approx l$, we obtain an explicit expression for the non-resonant PDI threshold in an inhomogeneous plasma,

$\left|E_{0 \perp}\right|^{2}>\frac{8 \pi L\left(\zeta_{i}\right)}{b_{e}^{3 / 4}} \frac{\omega_{p e}^{2}}{\omega_{U H}^{2}} \sqrt{A_{2}\left|\left(\frac{A_{2} \omega_{c e}^{2}}{A_{1} \omega_{U H}^{2}}+1\right) \frac{r_{L e}}{2 L_{N}}+\left(\frac{A_{2} \omega_{p e}^{2}}{A_{1} \omega_{U H}^{2}}+\frac{\omega_{c e}^{2}}{\omega_{p e}^{2}}\right) \frac{r_{L e}}{L_{B}}\right|} \frac{N_{e}^{(0)} T_{e}}{\epsilon_{0}}$.

Now that theoretical electric field PDI thresholds for decay of the cold X-mode pump wave into warm UH and LH daughter waves have been determined for both the resonant and non-resonant cases, we may determine the gyrotron power threshold near the UHR in the ASDEX Upgrade discharge considered earlier.

\section{Investigation of PDIs near the UHR in ASDEX Upgrade}

\subsection{Field Enhancement near the UHR}

The main problem in going from the electric field thresholds to gyrotron power thresholds is to connect $\left|E_{0}\right|$ to $P_{0}$ for various gyrotron settings at various points in the plasma. Away from the UHR, this may be done relatively simply if we assume the beam to have a narrow Gaussian profile around the CTS probe ray. With this assumption, we can evaluate all wave propagation related quantities on the CTS probe ray, and $\mathbf{E}_{0}$ may be written as,

$$
\mathbf{E}_{0}(\rho, s)=\left|E_{0}(0, s)\right| \mathrm{e}^{-\rho^{2} / W^{2}(s)} \mathbf{e}_{0}\left(\mathbf{r}(s), \mathbf{k}_{0}(s), \omega_{0}\right),
$$

where $\rho$ is the shortest distance to the CTS probe ray, occurring at the point $s$ along the CTS probe ray, while $W(s)$ and $\mathbf{e}\left(\mathbf{r}(s), \mathbf{k}_{0}(s), \omega_{0}\right)$ define the beam width and the geometric optics (X-mode) unit polarization vector at the point on the CTS probe ray characterised by $s$, respectively. The relation between 
$\left|E_{0}(0, s)\right|$ and $P_{0}$ is found by first taking the wave energy at a given $s$ to propagate at the group velocity of the corresponding point on the CTS probe ray, $\mathbf{v}_{g}\left(\mathbf{r}(s), \mathbf{k}_{0}(s), \omega_{0}\right)$. The total wave energy density, $U(\rho, s)$, is determined in a similar manner: $U(\rho, s)=\mathcal{M}\left(\mathbf{r}(s), \mathbf{k}_{0}(s), \omega_{0}\right)\left|E_{0}(\rho, s)\right|^{2}$, where $\mathcal{M}(\mathbf{r}, \mathbf{k}, \omega)=$ $\mathbf{e}_{0}^{*}(\mathbf{r}, \mathbf{k}, \omega) \cdot[\partial \mathbf{M}(\mathbf{r}, \mathbf{k}, \omega) / \partial \omega] \cdot \mathbf{e}_{0}(\mathbf{r}, \mathbf{k}, \omega)$, with $\mathbf{M}$ being the Maxwell operator from 51. Now, the intensity of the beam is $I(\rho, s)=U(\rho, s) v_{g}\left(\mathbf{r}(s), \mathbf{k}_{0}(s), \omega_{0}\right)=$ $\overline{\mathcal{M}}\left(\mathbf{r}(s), \mathbf{k}_{0}(s), \omega_{0}\right) v_{g}\left(\mathbf{r}(s), \mathbf{k}_{0}(s), \omega_{0}\right)\left|E_{0}(0, s)\right|^{2} \mathrm{e}^{-2 \rho^{2} / W^{2}(s)}$ and the total total power at a given $s, P(s)=\mathcal{F} \mathrm{e}^{-\tau(s)} P_{0}$, is found by the integral $P(s)=2 \pi \int_{0}^{\infty} \rho I(\rho, s) \mathrm{d} \rho$, assuming a large radius of curvature of the CTS probe ray compared with $W(s)$, from which

$$
\left|E_{0}(0, s)\right|=\sqrt{\frac{2 \mathcal{F} \mathrm{e}^{-\tau(s)} P_{0}}{\pi W^{2}(s) v_{g}\left(\mathbf{r}(s), \mathbf{k}_{0}(s), \omega_{0}\right) \mathcal{M}\left(\mathbf{r}(s), \mathbf{k}_{0}(s), \omega_{0}\right)}} .
$$

For electromagnetic waves in a vacuum, $v_{g}=c$ and $\mathcal{M}=\epsilon_{0} / 2$, 63) coincides with the well-known expression for a Gaussian beam; (63) also agrees with the result of [16] for propagation of a Gaussian beam parallel to the density gradient (and perpendicular to B) in a plasma slab, using $v_{g}$ and $\mathcal{M}$ for cold X-mode radiation. $W(s)$ is undetermined by the above discussion, but since we are only interested in an estimate of $\mathbf{E}_{0}$, we take it to be that of a free-space Gaussian beam, $W^{2}(s)=W_{0}^{2}+4 c^{2}\left(s-s_{0}\right)^{2} /\left(\omega_{0} W_{0}\right)^{2}$, with $W_{0}=2.29 \mathrm{~cm}$ and $s_{0}=85.4 \mathrm{~cm}$ (for $s=0$ at the gyrotron launch point) characterising the beam waist and focal point of the ASDEX Upgrade gyrotrons, respectively; more accurate values of $W(s)$, as well as $\mathbf{E}_{0}$ itself, away from the UHR may be obtained using beam tracing codes such as TORBEAM [53] or WKBeam [54. Note that the beam parameters are unchanged by the reflection from the high-field side wall, as it is modelled by a locally plane mirror.

(63) clearly shows field amplification at points with low group velocity, e.g. near the $\mathrm{UHR}$, and $\left|E_{0}\right|$ is additionally seen to be proportional to $\sqrt{\mathcal{F} \mathrm{e}^{-\tau(s)} P_{0}} / W(s)$, which is physically sensible. It does, however, just as clearly break down at the exact UHR, where $v_{g} \rightarrow 0$ and $\left|E_{0}(0, s)\right| \rightarrow \infty$, due to the failure of the geometric optics approximations at this point. Very close to the UHR we are thus forced to implement a full-wave solution in order to obtain meaningful results; this paper uses a simple 1D model, also used in [1], to estimate the field enhancement in the above region. The model, inspired by [15], is based on the cold plasma fluid equations, but includes collisional damping to limit $\left|E_{0}\right|$ at the UHR.

The procedure used for determining the field enhancement is as follows: first, the CTS probe ray is traced, from which $\mathcal{F}, \tau$, and $\mathbf{E}_{0}(0, s)$ (away from the UHR) are calculated. At a point close to the UHR, $\mathbf{r}\left(s_{f}\right)$, the ray tracing is stopped and the plasma parameters, used in the 1D full-wave treatment, are extracted along a line parallel to $\mathbf{k}_{0}\left(s_{f}\right)$ at this point. Finally, $\mathbf{E}_{0}(0, s)$ is calculated close to the UHR by solving the 1D full-wave equations for an X-mode wave and equating the amplitude to that obtained from ray tracing at $\mathbf{r}\left(s_{f}\right)$. Details of the implementation are found in [1]. Once the above quantities have been calculated, $\mathbf{E}_{0}(\rho, s)$ may be found from 62): $\mathbf{E}_{0}(\rho, s)=\mathbf{E}_{0}(0, s) \mathrm{e}^{-\rho^{2} / W^{2}(s)}$. 
It is clear that the above procedure contains a somewhat arbitrary step, namely the selection of the point at which the switch from ray tracing to the $1 \mathrm{D}$ full-wave solution is made and the length of the domain in which the 1D full-wave equations are solved. The choice of the transition point has some influence on the precise field profile near the UHR, but the exact domain length appears to be unimportant, so long as the UHR is present within the domain. The CTS probe ray may change direction rather abruptly at the transition between the ray tracing and the $1 \mathrm{D}$ full-wave regions, and no account can be made of the precise geometry and resulting refractive effects in the $1 \mathrm{D}$ full-wave model. Despite these shortcomings, the method is sufficient for obtaining the estimates in which we are interested. In the following, the transition between ray tracing and the $1 \mathrm{D}$ full-wave treatment is made at the same grid point of the X-mode ray (only changed for the peaked profile at $t=3.600 \mathrm{~s}$ ) and the length of the $1 \mathrm{D}$ full-wave domain is 3 $\mathrm{cm}$ (except at $t=\{3.600 \mathrm{~s}, 3.700 \mathrm{~s}\}$ for the peaked profiles, where it is $2.5 \mathrm{~cm}$ ). These parameters are chosen to ensure that the UHR is encountered and that the results are physically reasonable, i.e. avoiding artificial infinite field enhancements resulting from fast decay of the amplitude in the evanescent region and limited numerical accuracy.

Figure 8 contains logarithmic plots of $\left|E_{0 \perp}(0, s)\right| /\left|E_{0}(0,0)\right|$, calculated using the method described above, along the reflected X-mode ray at $t=2.900 \mathrm{~s}$ shown in figure 4 ( $s=0$ on the high-field side wall); these plots are illustrative of the field enhancement obtained in all cases. The left pane of figure 8 displays $\left|E_{0 \perp}(0, s)\right| /\left|E_{0}(0,0)\right|$ along the entire ray; apart from field enhancement near the UHR at $s \approx 20 \mathrm{~cm}$, it also shows the behaviour around the ECR at $s \approx 7 \mathrm{~cm}$. The right pane of figure 8 displays $\left|E_{0 \perp}(0, s)\right| /\left|E_{0}(0,0)\right|$ close to the UHR, and particularly shows the reduction of the pump wavelength (increase of $k_{0}$ ) as the UHR is approached, expected from cold plasma theory. A similar effect was not observed by [1] due to an error in the association of $\left|E_{0 \perp}\right|$ with $s$ in the $1 \mathrm{D}$ full-wave code used in that work. The transition between ray tracing and the 1D full-wave model is clearly visible in both panes, since the plane wave-like variation on the scale of the wavelength along the ray is factored out of $\mathbf{E}_{0}$ in the geometric optics approximation, while such a factorisation is generally not possible in the full-wave case and therefore not carried out; the amplitude of $\left|E_{0 \perp}(0, s)\right|$ does, however, vary relatively smoothly across the transition, as it should. A very large field enhancement, $\left|E_{0 \perp}(0, s)\right| /\left|E_{0}(0,0)\right| \approx 2 \times 10^{3}$, is obtained at the UHR (just before the evanescence region) in all cases, pointing to this being the maximum field enhancement allowed by collisions, which is confirmed by its reduction when the collision frequency increased keeping all other quantities constant.

\subsection{PDI Thresholds and Frequency Shifts}

Now that we have a model for $\left|E_{0 \perp}(0, s)\right|$ as a function of $P_{0}$ around the UHR, we proceed to determine the homogeneous and inhomogeneous thresholds for resonant and non-resonant PDIs using (51), (53), (59), and (61), with $b_{e}$ and $\zeta_{i}$ given by (44) and (45), respectively. The calculation is only carried out along the CTS probe ray, as the 


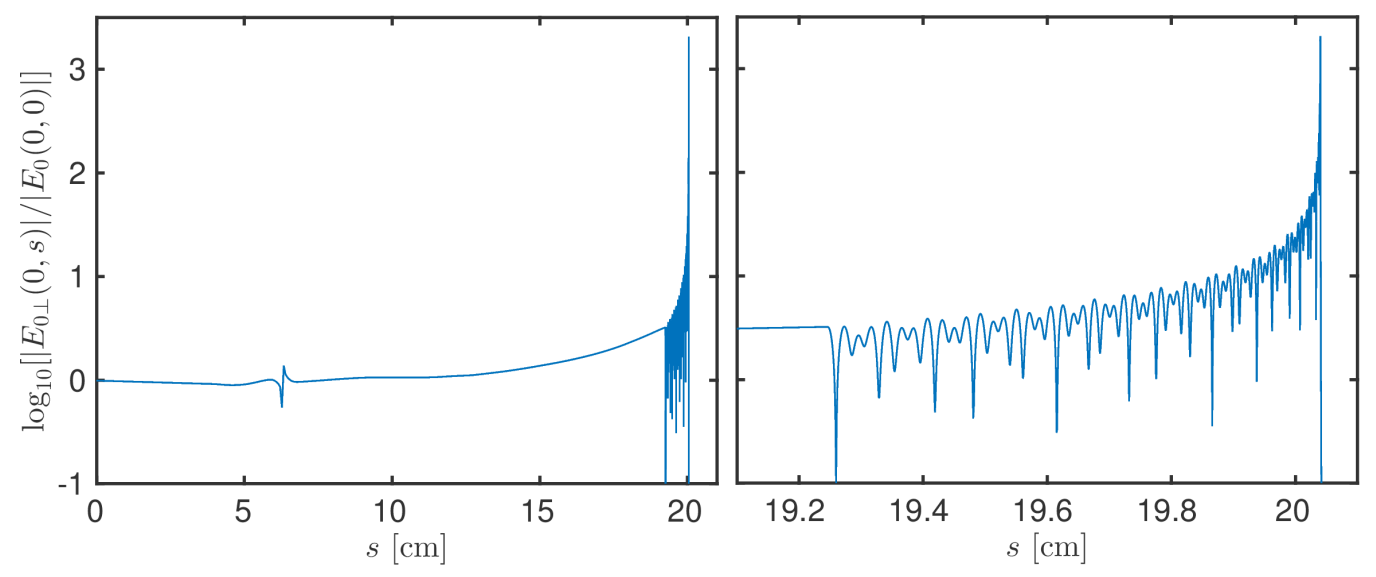

Figure 8. Logarithmic plots of the field enhancement along the ray of the reflected Xmode radiation for the peaked $N_{e}^{(0)}$ - and $T_{e}$-profiles at $t=2.900 \mathrm{~s}$ in ASDEX Upgrade discharge 28286 .

lowest thresholds are expected to occur along it. Since (51) and (53) are essentially identical in the region $L\left(\zeta_{i}\right) \sim 1$, where Eq. (51) is valid, only the homogeneous threshold given by (53) is plotted, where $\nu_{e}$ is identified with the electron(-ion) Coulomb collision frequency in a simple plasma given by [51]. $L_{N}$ and $L_{B}$ are calculated from the variation along the CTS probe ray, taking the angle between $\mathbf{k}_{0}$ and $\mathbf{B}$ into account, consistent with the treatment of the plasma parameter gradients in the $1 \mathrm{D}$ full-wave model [1]. The value of $\left|E_{0 \perp}\right|$ used in (53), (59) and (61) also needs some consideration due to the rapid variation of $\left|E_{0 \perp}\right|$ close to the UHR. We are modelling the PDI as occurring over a distance $l\left(s_{r}\right)$ around the point $s_{r}$ at which the selection rules are exactly satisfied, and since $G \propto\left|E_{0 \perp}\right|^{2}$ for both the resonant and non-resonant PDI in an inhomogeneous plasma, it is therefore appropriate use $\left|E_{0 \perp}\right|^{2}$ averaged over an interval of length $l\left(s_{r}\right)$ around $s_{r}$, denoted by $\left\langle\left|E_{0 \perp}\right|^{2}\right\rangle$, in $(59)$ and $(61) ;\left\langle\left|E_{0 \perp}\right|^{2}\right\rangle$ is also used in (53), as this threshold is mainly included to assess the importance of collisional damping on the PDI threshold. For simplicity, $\left\langle\left|E_{0 \perp}\right|^{2}\right\rangle$ is calculated in an interval $s \in\left[s_{r}-l\left(s_{r}\right) / 2, s_{r}+l\left(s_{r}\right) / 2\right]$ at each $s_{r}$ along the CTS probe ray. Once $\left\langle\left|E_{0 \perp}\right|^{2}\right\rangle$ is known for a given $P_{0}$, the gyrotron power threshold, $P_{0}^{\text {th }}$, is easily obtained using the fact that $\left\langle\left|E_{0 \perp}\right|^{2}\right\rangle \propto P_{0}: P_{0}^{\text {th }}=\left[\left\langle\left|E_{0 \perp}^{\text {th }}\right|^{2}\right\rangle /\left\langle\left|E_{0 \perp}\right|^{2}\right\rangle\right] P_{0}$, with $\left\langle\left|E_{0 \perp}^{\text {th }}\right|^{2}\right\rangle$ representing the right hand sides of (53) (homogeneous), (59) (inhomogeneous resonant) and (61) (inhomogeneous non-resonant); the resulting gyrotron power thresholds are denoted $P_{0}^{\text {th,hom }}, P_{0}^{\text {th,res }}$ and $P_{0}^{\text {th,nonres }}$, respectively.

Figure 9 shows logarithmic plots of $P_{0}^{\text {th,nonres }}, P_{0}^{\text {th,res }}$ and $P_{0}^{\text {th,hom }}$ in watt, as well as $\sqrt{\left\langle\left|E_{0 \perp}(0, s)\right|^{2}\right\rangle} /\left|E_{0}(0,0)\right|$, close to the UHR at $t=2.900 \mathrm{~s}$ for the ray in figure 4 ; it is illustrative of all cases. Evidently, $P_{0}^{\text {th,hom }}$ is always much lower than $P_{0}^{\text {th,nonres }}$ and $P_{0}^{\text {th,res }}$, which is necessary for the neglect of collisions in the calculation of these. As expected from the discussion of figure 7, the PDI is non-resonant in the considered ASDEX Upgrade equilibrium, with $P_{0}^{\text {th,nonres }}$ being almost two orders of magnitude larger than $P_{0}^{\text {th,res }}$. Further, $P_{0}^{\text {th,nonres }}, P_{0}^{\text {th,res }}$ and $P_{0}^{\text {th,hom }}$ increase slightly, while 


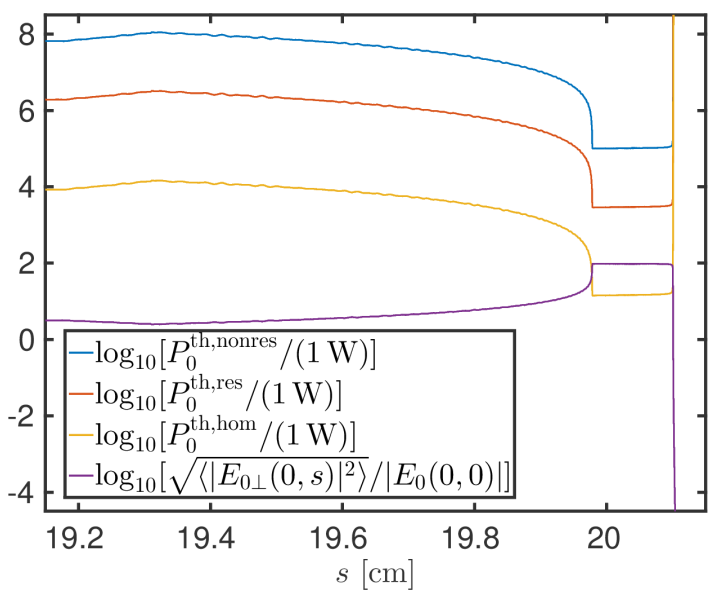

Figure 9. Logarithmic plots of the power thresholds and root mean square field enhancement along the ray of the reflected Xmode radiation for the peaked profile in ASDEX Upgrade discharge 28286 at $t=2.900 \mathrm{~s}$.

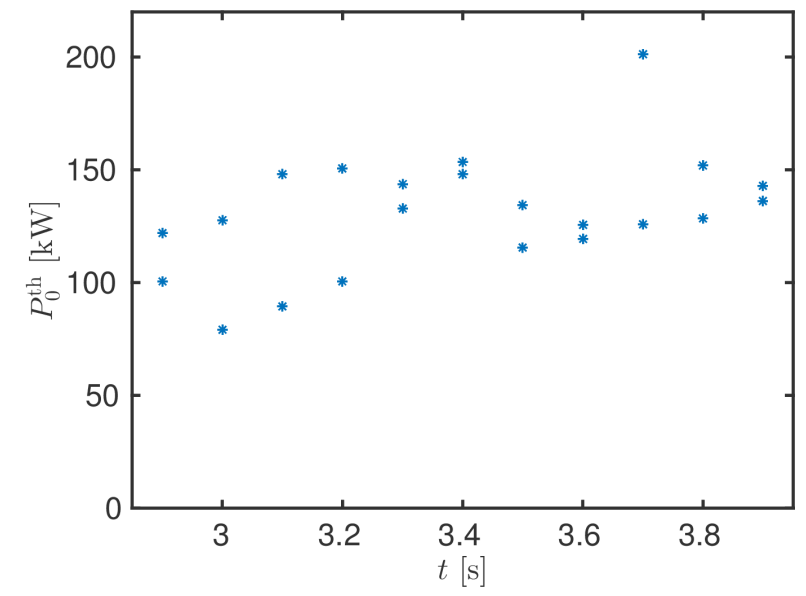

Figure 10. Gyrotron power threshold, $P_{0}^{\text {th }}$, versus $t$ in ASDEX Upgrade discharge 28286. $P_{0}^{\text {th }} \sim$ $100 \mathrm{~kW}$ in the shown part of the discharge and generally below the nominal gyrotron power, $P_{0}=$ $200 \mathrm{~kW}$.

$\sqrt{\left\langle\left|E_{0 \perp}(0, s)\right|^{2}\right\rangle} /\left|E_{0}(0,0)\right|$ decreases slightly, at the transition from ray tracing to the 1D full-wave model due to the inclusion of wave lobes (compare with figure 8) and all quantities display a flat region around the point of maximum field enhancement at the UHR; the lengths of the above features indicate that $l \sim 1 \mathrm{~mm}$ near the UHR. The minimum non-resonant gyrotron power threshold in figure 9 is $P_{0}^{\text {th }} \approx 100 \mathrm{~kW}$, which is on the order of the nominal experimental gyrotron power of $200 \mathrm{~kW}$. We thus expect the PDI to occur for the peaked $N_{e}^{(0)}$ - and $T_{e}$-profiles at $t=2.900 \mathrm{~s}$, allowing it to account for the peaks observed in figure 3 at this time point.

Figure 10 shows $P_{0}^{\text {th }}$, determined in a manner similar to that from figure 9, for $t \geq 2.900 \mathrm{~s}$. Clearly, $P_{0}^{\text {th }} \sim 100 \mathrm{~kW}$ in all cases, and the presented theory thus predicts the occurrence of a PDI in the analysed part of the discharge, where peaks are also observed in figure 3. In order to assess if it is reasonable to associate the observed peaks with the aforementioned PDI, we plot the predicted high-frequency daughter wave frequencies, $\omega_{0} \pm \omega_{1}=\omega_{0} \pm \omega_{L H} \sqrt{1+A_{1} b_{e}}$, along with the observed CTS spectra, for $t \geq 2.900 \mathrm{~s}$ in figure 11 , the LH frequencies at the points of $P_{0}^{\text {th }}$ are also plotted for reference. Evidently, the dipole PDI theory overestimates the peak frequency shift of approximately $850 \mathrm{MHz}$, giving values in the range $(1.18 \pm 0.03) \mathrm{GHz}$, while the simple estimate of the LH frequency underestimates it, giving values in the range (695 \pm 8$) \mathrm{MHz}$. The dipole PDI theory does, however, provide an upper bound on the frequency shift of the peak, and the LH frequency a lower bound. The latter point may be understood from the fact that warm LH waves have $\omega_{1} \geq \omega_{L H}$ according to their dispersion relation, (41), while the former may be understood if we assume the warm UH wave to be forward scattered by the pump wave, following a line of inquiry similar to that of [5], such that $k_{1}=\left|k_{2}-k_{0}\right|$ (and hence the relevant $b_{e}$ ) is maximised in the dipole limit, where $k_{0}=0$, 

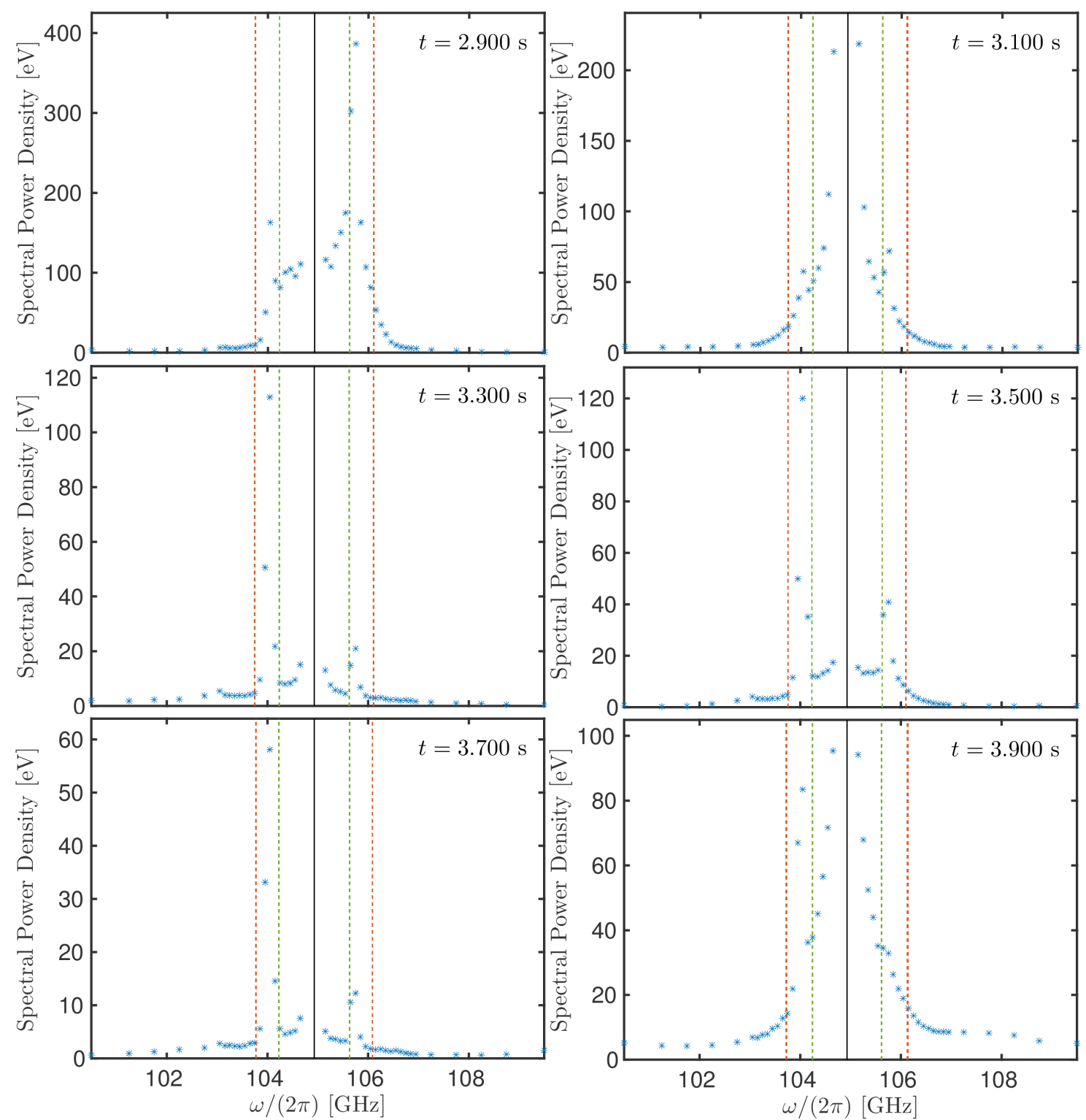

Figure 11. Predicted high-frequency daughter wave frequencies (orange dashed lines) and the gyrotron frequency shifted by the LH frequencies at the points of $P_{0}^{\text {th }}$ (green dashed lines), along with the experimental CTS power spectra for $t \geq 2.900 \mathrm{~s}$ in ASDEX Upgrade discharge 28286.

assuming $k_{2}>k_{0}$. The above considerations indicate that it is reasonable to associate the observed peaks with parametric decay of the (reflected) X-mode pump wave into a warm UH wave and a warm LH wave. Additionally, the upper and lower bounds on the frequency shift of the PDI peaks are important for the design of notch filters to protect millimetre-wave diagnostics against these. However, in order to determine the precise frequency shift of the peak top, a theory of the above PDI taking non-zero $\mathbf{k}_{0}$, as well as possible deviations from the resonant warm LH wave dispersion relation, into account is necessary. 


\section{Conclusions and Outlook}

In this paper we have investigated parametric decay of an electromagnetic pump wave into two electrostatic daughter waves, particularly an X-mode pump wave decaying into a warm UH wave and a warm LH wave. This PDI has been shown to occur for $\omega_{p e}<\sqrt{3}\left|\omega_{c e}\right|$, and analytical $\left|E_{0 \perp}\right|^{2}$-thresholds have been derived for homogeneous and inhomogeneous plasmas in the resonant and non-resonant cases. The theory has been applied to CTS experiments at ASDEX Upgrade where $105 \mathrm{GHz}$ O-mode radiation is injected from the low-field side, reflected by the high-field side wall, and part of the reflected radiation is coupled back into the plasma in X-mode, reaching the UHR where the above PDI may occur. The gyrotron power threshold of the above PDI is estimated to be $\sim 100 \mathrm{~kW}$, and is generally below the nominal gyrotron power of $200 \mathrm{~kW}$ used in the CTS experiment, indicating that the above PDI does indeed occur. This is further supported by the fact that the frequency shifts of the observed sidebands are reasonably close to the LH frequency and the value predicted by dipole PDI theory, which serve as lower and upper bounds of the frequency shifts, respectively. We note that the fraction of power reaching the UHR in X-mode in the experiment is a rather strong function of the toroidal gyrotron injection angle, peaking at approximately 0.04 close to the experimental value of $-5^{\circ}$, and falling to virtually zero for poloidal injection and toroidal injection angles numerically greater than $10^{\circ}$; both these regimes allow the PDI to be suppressed. PDI suppression at virtually poloidal injection is caused by a negligible fraction of reflected power being coupled to the plasma in X-mode, meaning that it should in principle always be possible to achieve PDI suppression by this method, but also that the method will only be applicable to narrow gyrotron beams of small angular divergence. PDI suppression at large toroidal injection angles relies on the ECR becoming optically thick, which requires $B$ to be large enough for the ECR to be located between the high-field side wall and the UHR, ideally inside the last closed flux surface. The latter method sets fewer restrictions on the gyrotron beam and has been successfully applied to suppress the PDI in $105 \mathrm{GHz}$ O-mode CTS at ASDEX Upgrade; it does, however, restrict the $B$ operation space. It is stressed that the PDI only occurs at the modest power levels of the reflected X-mode radiation $(\sim 10 \mathrm{~kW})$ due to strong field enhancement close to the UHR which may be determined by a full-wave calculation.

There are several lines of inquiry which warrant further investigation. We have employed the dipole approximation, $\mathbf{k}_{0} \approx \mathbf{0}$, in deriving the PDI growth rates, which appears to be the cause of the discrepancy between the predicted and observed PDI frequency shifts, and an investigation involving non-zero $\mathbf{k}_{0}$ is thus of interest; related to this, a treatment accounting for the deviation from the resonant LH dispersion relation (41) in the non-resonant case is also of interest. Further, it is of interest to repeat the PDI analysis for magnetised ions, with $\chi_{\sigma}(\mathbf{k}, \omega)$ from $(9)$ and $\nu_{\sigma} \approx 0$, corresponding to general pure ion Bernstein low-frequency daughter waves. Another point of interest is to investigate the sidebands observed during CTS experiments at LHD [7,8] in order to 
determine whether or not they may be explained by the theory employed to explain the sidebands during CTS experiments at ASDEX Upgrade. Additionally, confirmation of a gyrotron power threshold and the existence of warm LH daughter waves, along with ion heating generated by these [31-34], during CTS experiments at ASDEX Upgrade would provide further evidence for the theory; experiments of this type are planned for the 2017/2018 ASDEX Upgrade experimental campaign. The geometric optics and 1D full-wave models used to estimate $\mathbf{E}_{0}$ should also be compared with a full-wave solution taking the real geometry into account in order to assess their validity; such a full-wave solution would further allow us to investigate the cases in which the CTS probe ray does not reach to UHR in the main plasma, but where a significant fraction of the beam power may still do so. Finally, it would be of interest to perform fully nonlinear simulations of an X-mode beam encountering the UHR, e.g. using a particle-in-cell code like EPOCH [55], as done by [27] for a piecewise linear slab geometry, but employing a more realistic plasma profile in order to assess the actual frequency shift, growth rate and saturation level of the PDI in different experiments.

\section{Acknowledgements}

We thank Dr. A Köhn for helpful discussions about field enhancement of X-mode radiation near the UHR. This work was supported by a research grant (15483) from VILLUM FONDEN. This work has been carried out within the framework of the EUROfusion Consortium and has received funding from the Euratom research and training programme 2014-2018 under grant agreement No 633053. The views and opinions expressed herein do not necessarily reflect those of the European Commission.

\section{References}

[1] Hansen S K 2016 Parametric Decay and Anomalous Scattering from Tokamak Plasmas Master's thesis Technical University of Denmark Kgs. Lyngby URL http://www.staff.dtu.dk/skni/ $\sim /$ media/skni/SKH_Msc.ashx

[2] Porkolab M 1982 Parametric decay instabilities in ECR heated plasmas Proc. 2nd Workshop Hot Electron Ring Physics (San Diego) vol 1 ed N A Uckan (Alexandria: National Technical Information Service) p 237

[3] McDermott F S, Bekefi G, Hackett K E, Levine J S and Porkolab M 1982 Phys. Fluids 251488

[4] Tzoar N 1969 Phys. Rev. 178356

[5] Dodin I Y and Arefiev A V 2017 Phys. Plasmas 24032119

[6] Nielsen S K, Michelsen P K, Hansen S K, Korsholm S B, Leipold F, Rasmussen J, Salewski M, Schubert M, Stejner M, Stober J, Wagner D and the ASDEX Upgrade team 2017 Phys. Scr. 92 024001

[7] Nishiura M, Kubo S, Tanaka K, Seki R, Osagawa S, Shimozuma T, Okada K, Kobayashi S, Mutoh T, Kawahata K, Watari T, LHD Experiment Group, Saito T, Tatematsu Y, Korsholm S B and Salewski M 2014 Nucl. Fusion 54023006

[8] Kubo S, Nishiura M, Tanaka K, Moseev D, Ogasawara S, Shimozuma T, Yoshimura Y, Igami H, Takahashi H, Tsujimura T I, Makino R and LHD experiment group 2016 J. Instrum. 11 C06005

[9] Silin V P 1965 Sov. Phys. - JETP 211127

[10] Aliev Yu M, Silin V P and Watson C 1966 Sov. Phys. - JETP 23626 
[11] Amano T and Okamoto M 1969 J. Phys. Soc. Jpn. 26529

[12] Piliya A D 1971 Decay instability in weakly inhomogeneous plasma Proc. 10th Int. Conf. Phenomena in Ionized Gases (Oxford) ed R N Franklin (Oxford: Donald Parsons \& Co. Ltd.) p 320

[13] Rosenbluth M N 1972 Phys. Rev. Lett. 29565

[14] Piliya A D 1973 Sov. Phys. - JETP 37629

[15] White R B and Chen F F 1974 Plasma Phys. 16565

[16] Gusakov E Z and Popov A Yu 2016 Phys. Plasmas 23082503

[17] Gusakov E Z and Surkov A V 2007 Plasma Phys. Control. Fusion 49631

[18] Ott E, Hui B and Chu K R 1980 Phys. Fluids 231031

[19] Stefan V and Bers A 1984 Phys. Fluids 27175

[20] Kasymov Zh Zh, Näslund E, Starodub A N and Stenflo L 1985 Phys. Scr. 31201

[21] Sharma R P and Shukla P K 1983 Phys. Fluids 2687

[22] Murtaza G and Shukla P K 1984 J. Plasma Phys. 31423

[23] Leyser T B, Thidé B, Waldenvik M, Veszelei E, Frolov V L, Grach S M and Komrakov G P 1994 J. Geophys. Res. 9919555

[24] Lin A T and Lin C-C 1981 Phys. Rev. Lett. 4798

[25] Eliasson B and Shukla P K 2003 Phys. Lett. A 31291

[26] Xiang N and Cary J R 2011 Phys. Plasmas 18122107

[27] Arefiev A V, Dodin I Y, Köhn A, Du Toit E J, Holzhauer E, Shevchenko V F and Vann R G L 2017 Nucl. Fusion $\mathbf{5 7} 116024$

[28] Hiroe S and Ikegami H 1967 Phys. Rev. Lett. 191414

[29] Grek B and Porkolab M 1973 Phys. Rev. Lett. 30836

[30] Okabayashi M, Chen K and Porkolab M 1973 Phys. Rev. Lett. 311113

[31] Bulyginsky D G, Gusev V K, Djachenko V V, Irzak M A, Kantor M Yu, Larionov M M, Levin L S, Serebreny G A and Shustova N V 1984 ECR-heating of plasma in FT-1 tokamak and its influence on the ion component Proc. 11th European Conf. Controlled Fusion and Plasma Physics (Aachen) vol 1 (Mulhouse: European Physical Society) p 457

[32] Pietrzyk Z A, Pochelon A, Behn R, Bondeson A, Dutch M, Goodman T P, Tran M Q and Whaley D R 1993 Nucl. Fusion 33197

[33] Wilhelm R, Erckmann V, Janzen G, Kasparek W, Müller G, Räuchle E, Schüller P G, Schwörer K, Thumm M and the W VII-A team 1984 Plasma Phys. Control. Fusion 261433

[34] Erckmann V, Janzen G, Kasparek W, Müller G, Räuchle E, Schüller P G, Schwörer K, Thumm M, Wilhelm R and the W VII-A team 1985 Wave absorption and transport studies on ECR-heated stellarator plasmas in W VII-A Proc. 10th Int. Conf. Plasma Physics and Controlled Nuclear Fusion Research (London) vol 2 (Vienna: International Atomic Energy Agency) p 419

[35] Porkolab M 1978 Nucl. Fusion 18367

[36] Erckmann V and Gasparino U 1994 Plasma Phys. Control. Fusion 361869

[37] Laqua H P, Erckmann V, Hartfuß H J, Laqua H, W7-AS Team and ECRH Group 1997 Phys. Rev. Lett. 783467

[38] Laqua H P 2007 Plasma Phys. Control. Fusion 49 R1

[39] Shevchenko V, Cunningham G, Gurchenko A, Gusakov E, Lloyd B, O’Brien M, Saveliev A, Surkov A, Volpe F and Walsh M 2007 Fusion Sci. Technol. 52202

[40] Porkolab M 1974 Phys. Fluids 171432

[41] Opher M, Morales G J and Leboeuf J N 2002 Phys. Rev. E 66016407

[42] Porkolab M and Cohen B I 1988 Nucl. Fusion 28239

[43] Drake J F, Kaw P K, Lee Y C, Schmidt G, Liu C S and Rosenbluth M N 1974 Phys. Fluids 17 778

[44] Sagdeev R Z and Galeev A A 1969 Nonlinear Plasma Theory (New York: W. A. Benjamin, Inc.)

[45] Weiland J and Wilhelmsson H 1977 Coherent Non-Linear Interaction of Waves in Plasmas (Oxford: Pergamon Press) 
[46] Berger R L, Chen L, Kaw P K and Perkins F W 1977 Phys. Fluids 201864

[47] Bindslev H 1991 Plasma Phys. Control. Fusion 331775

[48] Bindslev H 1992 Plasma Phys. Control. Fusion 341601

[49] Fischer R, Fuchs C J, Kurzan B, Suttrop W, Wolfrum E and the ASDEX Upgrade team 2010 Fusion Sci. Technol. $\mathbf{5 8} 675$

[50] McCarthy P J, Martin P and Schneider W 1999 The CLISTE interpretive equilibrium code Tech. Rep. IPP 5/85 Max-Planck-Institut für Plasmaphysik Garching bei München

[51] Swanson D G 2003 Plasma Waves 2nd ed (Bristol: IOP Publishing)

[52] Verdon A L, Cairns I H, Melrose D B and Robinson P A 2009 Phys. Plasmas 16052105

[53] Poli E, Peeters A G and Pereverzev G V 2001 Comput. Phys. Commun. 13690

[54] Weber H, Maj O and Poli E 2015 EPJ Web Conf. 8701002

[55] Arber T D, Bennett K, Brady C S, Lawrence-Douglas A, Ramsay M G, Sircombe N J, Gillies P, Evans R G, Schmitz H, Bell A R and Ridgers C P 2015 Plasma Phys. Control. Fusion 57 113001 